\title{
Chiral states in bilayer graphene: Magnetic field dependence and gap opening
}

\author{
M. Zarenia, ${ }^{1}$ J. M. Pereira Jr., ${ }^{2}$ G. A. Farias, ${ }^{2}$ and F. M. Peeters ${ }^{1,2}$ \\ ${ }^{1}$ Department of Physics, University of Antwerp, Groenenborgerlaan 171, B-2020 Antwerpen, Belgium \\ ${ }^{2}$ Departamento de Física, Universidade Federal do Ceará, Fortaleza, Ceará, 60455-760, Brazil
}

(Received 19 May 2011; published 30 September 2011)

\begin{abstract}
At the interface of electrostatic potential kink profiles, one-dimensional chiral states are found in bilayer graphene (BLG). Such structures can be created by applying an asymmetric potential to the upper and the lower layers of BLG. We found the following: (i) due to the strong confinement by the single kink profile, the unidirectional states are only weakly affected by a magnetic field; (ii) increasing the smoothness of the kink potential results in additional bound states, which are topologically different from those chiral states; and (iii) in the presence of a kink-antikink potential, the overlap between the oppositely moving chiral states results in the appearance of crossing and anticrossing points in the energy spectrum. This leads to the opening of tunable minigaps in the spectrum of the unidirectional topological states.
\end{abstract}

DOI: 10.1103/PhysRevB.84.125451

PACS number(s): 71.10.Pm, 73.21.-b, 81.05.ue

\section{INTRODUCTION}

Carbon-based electronic structures have been the focus of intense research since the discovery of fullerenes and carbon nanotubes. ${ }^{1}$ More recently, the production of atomic layers of hexagonal carbon (called graphene) has renewed that interest, with the observation of striking mechanical and electronic properties, as well as ultrarelativisticlike phenomena in this condensed matter system. ${ }^{2,3}$ In that context, bilayer graphene (BLG), which is a system with two Van der Waals coupled sheets of graphene, has been shown to have features that make it a possible substitute of silicon in microelectronic devices. The carrier dispersion of pristine BLG is gapless and approximately parabolic at two points in the Brillouin zone $\left(K\right.$ and $\left.K^{\prime}\right) .{ }^{4}$ However, it was found that the application of perpendicular electric fields produced by external gates deposited on the BLG surface can induce a gap in the spectrum by creating a charge imbalance between the two graphene layers. ${ }^{5,6}$ The tailoring of the gap by an external field may be particularly useful for the development of devices. ${ }^{7,8}$ It was recently recognized that a tunable energy gap in BLG can allow the observation of new confined electronic states, which could be obtained by applying a spatially varying potential profile to create a position-dependent gap analogous to semiconductor heterojunctions.

An alternative way to create one-dimensional (1D) localized states in BLG has recently been suggested by Martin et al. ${ }^{9}$ and relies on the creation of a potential kink by an asymmetric potential profile (see Fig. 1). Such kink potential can also be realized in $p$ - $n$ junctions. They showed that localized chiral states arise at the location of the kink, with energies inside the energy gap. These states correspond to unidirectional motion of electrons, which are analogous to the edge states in a quantum Hall system. From a practical standpoint, the kinks may be envisaged as configurable metallic nanowires embedded in a semiconductor medium. Moreover, the carrier states in this system are expected to be robust with regard to scattering and may display Luttinger liquid behavior. ${ }^{10}$

An additional tool for the manipulation of charged states in BLG is the use of magnetic fields. The application of an external magnetic field perpendicular to the BLG sheet causes the appearance of Landau levels, which can be significantly modified by the induced gap, leading to effects such as the lifting of valley degeneracy caused by the breaking of the inversion symmetry by the electrostatic bias. ${ }^{11-13}$ Recently the transport properties of $p-n-p$ junctions in bilayer graphene were experimentally investigated in the presence of a perpendicular magnetic field. ${ }^{14}$

In this paper, we generalize previous work on topological confinement in bilayer graphene on three levels: (i) we investigate the effect of smoothing the kink potential on the topological states; (ii) the effect of a perpendicular magnetic field is studied; and (iii) we investigate a new system that consists of a coupled kink-antikink structure. We demonstrate that the latter opens a gap in the $1 \mathrm{D}$ electron states. The paper is organized as follows. In Sec. II, we present the theoretical formalism. The results for a single kink potential profile are discussed in Secs. III A and III B. In Secs. IV A and IV B, we show the results for the kink-antink potential, respectively, for zero and nonzero magnetic fields. Finally, we conclude the remarks of the paper in Sec. V.

\section{MODEL}

We employ a two-band continuum model to describe the BLG sheet. In this model, the system is described by four sublattices, two in the upper $(A, B)$ and two in the lower ( $A^{\prime}$ and $B^{\prime}$ ) layer. $^{7}$ The interlayer coupling is given by the hopping parameter $t \approx 400 \mathrm{meV}$ between sites $A$ and $B^{\prime}$. The Hamiltonian around the $K$ valley of the first Brillouin zone can be written as

$$
H=-\frac{1}{t}\left[\begin{array}{cc}
0 & \left(\pi^{\dagger}\right)^{2} \\
(\pi)^{2} & 0
\end{array}\right]+\left[\begin{array}{cc}
U(x) & 0 \\
0 & -U(x)
\end{array}\right],
$$

where $\pi=v_{F}\left(p_{x}+i p_{y}\right), p_{x, y}=-i \hbar \partial_{x, y}+e A_{x, y}$ is the momentum operator in the presence of an external magnetic field with $A_{x, y}$ being the components of the vector potential $\mathbf{A}$, $v_{F}=10^{6} \mathrm{~m} / \mathrm{s}$ is the Fermi velocity, $U(x)$ and $-U(x)$ are the electrostatic potentials, respectively, applied to the upper and lower layers. The eigenstates of the Hamiltonian Eq. (1) are two-component spinors $\Psi(x, y)=\left[\psi_{a}(x, y), \psi_{b}(x, y)\right]^{T}$, where $\psi_{a, b}$ are the envelope functions associated with the probability amplitudes at sublattices $A$ and $B^{\prime}$ at the respective layers of the BLG sheet. Since $\left[H, p_{y}\right]=0$, the momentum 
(a)

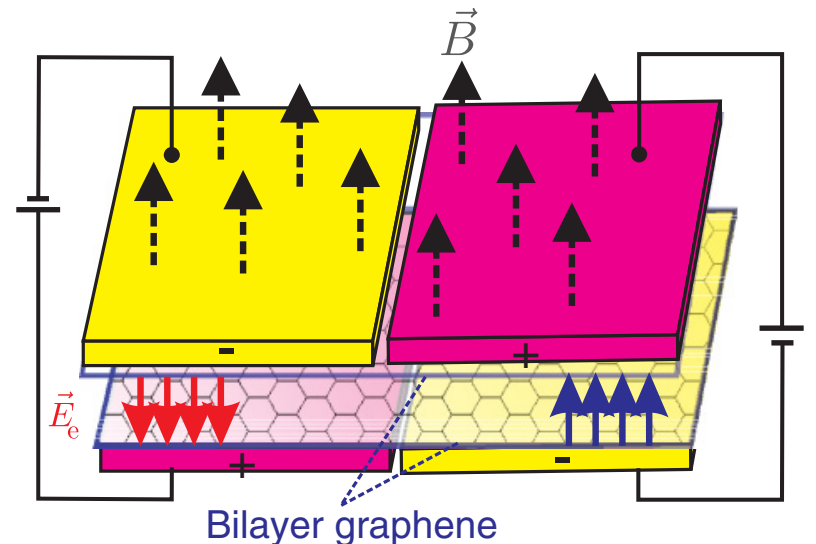

(b)

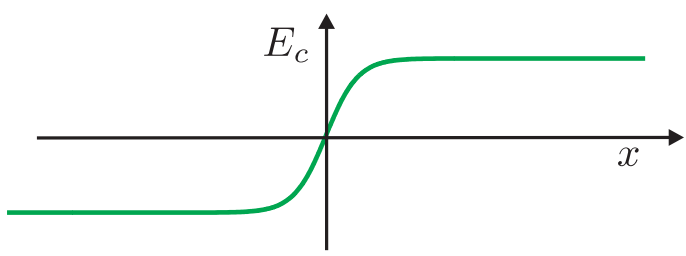

(c)

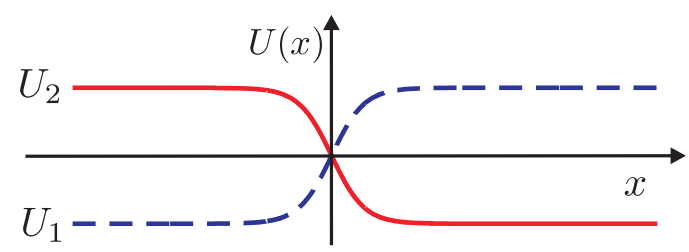

FIG. 1. (Color online) (a) Schematic illustration of the nonuniformly gated bilayer graphene device for the creation of a kink potential. Applied gated voltage to the upper and lower layers with opposite sign induces an electric field $\boldsymbol{E}_{\boldsymbol{e}}$, with preferential direction. An external magnetic field $\boldsymbol{B}=B \hat{z}$ is applied perpendicular to the bilayer graphene sheets. (b) Electric field between the two graphene layers. (c) Potential on layer $1\left(U_{1}\right)$ and layer $2\left(U_{2}\right)$.

along the $y$ direction is a conserved quantity and, therefore, we can write

$$
\psi(x, y)=e^{i k_{y} y}\left[\varphi_{a}(x), \varphi_{b}(x)\right]^{T},
$$

where $k_{y}$ is the wave vector along the $y$ direction.

In order to apply a perpendicular magnetic field to the bilayer sheet, we employ the Landau gauge for the vector potential $\mathbf{A}=(0, B x, 0)$. The Hamiltonian (1) acts on the wave function of Eq. (2), which leads to the following coupled second-order differential equations:

$$
\begin{aligned}
& {\left[\frac{\partial}{\partial x^{\prime}}+\left(k_{y}^{\prime}+\beta x^{\prime}\right)\right]^{2} \varphi_{b}=\left[\epsilon-u\left(x^{\prime}\right)\right] \varphi_{a},} \\
& {\left[\frac{\partial}{\partial x^{\prime}}-\left(k_{y}^{\prime}+\beta x^{\prime}\right)\right]^{2} \varphi_{a}=\left[\epsilon+u\left(x^{\prime}\right)\right] \varphi_{b},}
\end{aligned}
$$

where, in the above equations, we used dimensionless units $x^{\prime}=x / l, k_{y}^{\prime}=k_{y} l, \epsilon=E / t$, and $u\left(x^{\prime}\right)=U(x) / t$, where $l=$ $\hbar v_{F} / t=1.6455 \mathrm{~nm}, \beta=(e B / \hbar) l^{2}(=0.0041$ for $B=1) \mathrm{T}$. The steplike kink [see Fig. 1(c)] is modeled by

$$
u\left(x^{\prime}\right)=u_{b} \tanh \left(x^{\prime} / \delta\right),
$$

where $u_{b}$ is the maximum value of the gate voltage, in dimensionless unit, in each BLG layer. Here, $\delta$ denotes the width of the region in which the potential switches its sign in each layer. This parameter is determined by the distance between the gates used to create the gap. Next, we numerically solve Eqs. (3) to obtain the dependence of the energy levels on the magnetic field and potential parameters. For the case of a sharp kink potential $\delta \rightarrow 0$ and in the absence of a magnetic field, i.e., $B=0$, Eqs. (3) reduce to

$$
\begin{aligned}
& {\left[\frac{\partial}{\partial x^{\prime}}+k_{y}^{\prime}\right]^{2} \varphi_{b}=\left[\epsilon-u\left(x^{\prime}\right)\right] \varphi_{a},} \\
& {\left[\frac{\partial}{\partial x^{\prime}}-k_{y}^{\prime}\right]^{2} \varphi_{a}=\left[\epsilon+u\left(x^{\prime}\right)\right] \varphi_{b},}
\end{aligned}
$$

where $u\left(x^{\prime}\right)=u_{b}\left[\Theta\left(x^{\prime}\right)-\Theta\left(-x^{\prime}\right)\right]$. We simply decouple Eqs. (5) and obtain

$$
\left[\frac{\partial^{2}}{\partial x^{\prime 2}}+\lambda_{ \pm}^{2}\right] \varphi_{a}=0
$$
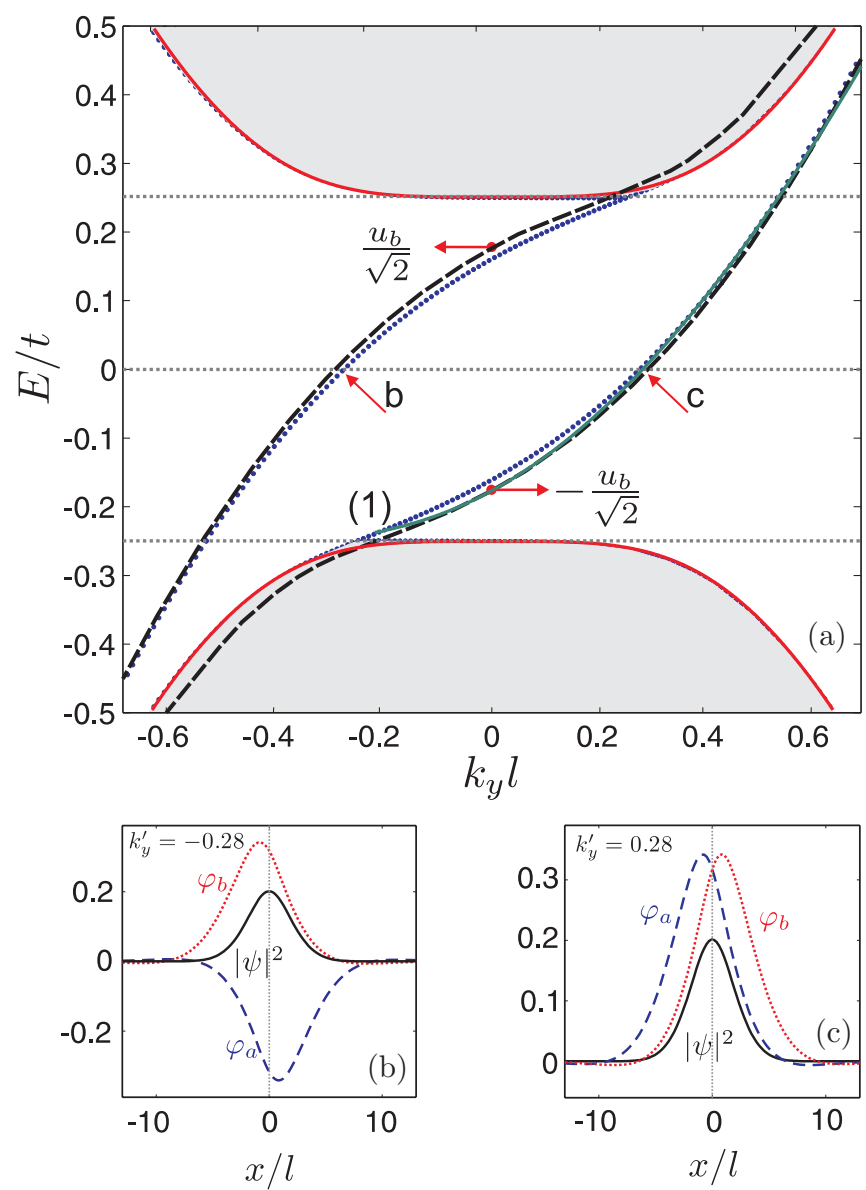

FIG. 2. (Color online) (a) Energy levels for a single kink profile on bilayer graphene with $u_{b}=0.25$. Dotted curves are the numerical results for $\delta=1$ and dashed curves are the analytical results for $\delta=0$ using Eq. (8). The solid red curves are the energy levels of a biased BLG. The solid green curve [indicated by the symbol (1)] shows a fitted function to the numerical results. The lower panels show the wave spinors and probability density corresponding to the states that are indicated by the arrows b and $\mathrm{c}$ in panel (a). 
where $\lambda_{ \pm}=\left[-k_{y}^{\prime 2} \pm \sqrt{\epsilon^{2}-u_{b}^{2}}\right]^{1 / 2}$, which can be a complex quantity. The solutions for $x^{\prime}<0\left(\psi^{<}\right)$and $x^{\prime} \geqslant 0\left(\psi^{>}\right)$are given by

$$
\begin{gathered}
\psi\left(x^{\prime}\right)_{ \pm}^{<}=\left(\begin{array}{c}
e^{i \lambda_{ \pm} x^{\prime}} \\
f_{ \pm} e^{i \lambda_{ \pm} x^{\prime}}
\end{array}\right), \\
\psi\left(x^{\prime}\right)_{ \pm}^{>}=\left(\begin{array}{c}
e^{-i \lambda_{ \pm} x^{\prime}} \\
g_{ \pm} e^{-i \lambda_{ \pm} x^{\prime}}
\end{array}\right),
\end{gathered}
$$

where $\quad f_{ \pm}=\left(i \lambda_{ \pm}-k_{y}^{\prime}\right)^{2} /\left(\epsilon-u_{b}\right) \quad$ and $\quad g_{ \pm}=\left(i \lambda_{ \pm}+\right.$ $\left.k_{y}^{\prime}\right)^{2} /\left(\epsilon+u_{b}\right)$. The above solutions should satisfy the asymptotics $\varphi_{a, b}^{>}\left(x^{\prime} \rightarrow \infty\right)=0$ and $\varphi_{a, b}^{<}\left(x^{\prime} \rightarrow-\infty\right)=0$. Matching the solutions and the first derivatives at $x^{\prime}=0$ gives a homogeneous set of algebraic equations, which in matrix form become

$$
\left(\begin{array}{cccc}
1 & 1 & -1 & -1 \\
f_{+} & f_{-} & -g_{+} & -g_{-} \\
\lambda_{+} & \lambda_{-} & \lambda_{+} & \lambda_{-} \\
f_{+} \lambda_{+} & f_{-} \lambda_{-} & g_{+} \lambda_{+} & g_{-} \lambda_{-}
\end{array}\right)\left(\begin{array}{l}
C_{1} \\
C_{2} \\
C_{3} \\
C_{4}
\end{array}\right)=0
$$

Solutions are found when the determinant of the matrix is set to zero, from which we obtain the energy spectrum. Notice that Eq. (8) leads to four solutions, of which two of them, i.e., $\pm u_{b}$, do not satisfy Eqs. (5) and are not acceptable. In the limiting case $\epsilon \ll u_{b}$, we are able to obtain an analytical expression for the energy

$$
\begin{aligned}
\epsilon_{ \pm}= & \frac{u_{b}}{\alpha}\left\{4 k_{y}^{\prime} \sqrt{\epsilon_{0}}\left[u_{b} \sin (\theta / 2)+k_{y}^{\prime 2} \cos (\theta / 2)\right]\right. \\
& \left. \pm\left[56 k_{y}^{\prime 8}+14 u_{b}^{4}+70 u_{b}^{2} k_{y}^{\prime 4}-k_{y}^{\prime 2} \epsilon_{0}\left(40 k_{y}^{\prime 4}+46 u_{b}^{2}\right)\right]^{1 / 2}\right\}
\end{aligned}
$$

where $\epsilon_{0}=\sqrt{k_{y}^{\prime 4}+u_{b}^{2}}, \quad \alpha=6 k_{y}^{\prime 4}+7 u_{b}^{2}-6 k_{y}^{\prime 2} \epsilon_{0}, \quad$ and $\theta=$ $\tan ^{-1}\left(u_{b} / k_{y}^{\prime 2}\right)$. Solving the above equation for $\epsilon=0$, we find that $k_{y}^{\prime}= \pm \sqrt{u_{b} / \sqrt{8}}\left(\approx 0.3\right.$ for $\left.u_{b}=0.25\right)$.

Next, we consider a sharp kink potential in parallel with an antikink potential that are located at $x^{\prime}=-d$ and $x^{\prime}=+d$. In this case, we have to consider three regions, i.e., $x^{\prime}>d$ $\left[\psi_{\mathrm{I}}\left(x^{\prime}\right)\right],-d \leqslant x^{\prime} \leqslant-d\left[\psi_{\mathrm{II}}\left(x^{\prime}\right)\right]$, and $x^{\prime}>d\left[\psi_{\mathrm{III}}\left(x^{\prime}\right)\right]$, and the solutions are given by

$$
\begin{aligned}
& \psi_{\mathrm{I}}\left(x^{\prime}\right)_{ \pm}=\left(\begin{array}{c}
e^{i \lambda_{ \pm} x^{\prime}} \\
g_{ \pm} e^{i \lambda_{ \pm} x^{\prime}}
\end{array}\right), \\
& \psi_{\mathrm{II}}\left(x^{\prime}\right)_{ \pm}=\left(\begin{array}{c}
e^{ \pm i \lambda_{ \pm} x^{\prime}} \\
f_{ \pm} e^{i \lambda_{ \pm} x^{\prime}}
\end{array}\right), \\
& \psi_{\mathrm{III}}\left(x^{\prime}\right)_{ \pm}=\left(\begin{array}{c}
e^{-i \lambda_{ \pm} x^{\prime}} \\
g_{ \pm} e^{-i \lambda_{ \pm} x^{\prime}}
\end{array}\right) .
\end{aligned}
$$

Matching the solutions and their first derivatives at $x^{\prime}= \pm d$ leads to a set of eight algebraic equations, which in matrix form becomes

$$
\left(\begin{array}{cccccccc}
\kappa_{+}^{-} & \kappa_{-}^{-} & -\kappa_{+}^{-} & -\kappa_{-}^{-} & -\kappa_{+}^{+} & -\kappa_{-}^{+} & 0 & 0 \\
h_{+} \kappa_{+}^{-} & h_{-} \kappa_{-}^{-} & -f_{+} \kappa_{+}^{-} & -f_{-} \kappa_{-}^{-} & -l_{+} \kappa_{+}^{+} & -l_{-} \kappa_{-}^{+} & 0 & 0 \\
\lambda_{+} \kappa_{+}^{-} & \lambda_{-} \kappa_{-}^{-} & -\lambda_{+} \kappa_{+}^{-} & -\lambda_{-} \kappa_{-}^{-} & \lambda_{+} \kappa_{+}^{+} & \lambda_{-} \kappa_{-}^{+} & 0 & 0 \\
h_{+} \lambda_{+} \kappa_{+}^{-} & h_{-} \lambda_{-} \kappa_{-}^{-} & -f_{+} \lambda_{+} \kappa_{+}^{-} & -f_{-} \lambda_{-} \kappa_{-}^{-} & l_{+} \lambda_{+} \kappa_{+}^{+} & l_{-} \lambda_{-} \kappa_{-}^{+} & 0 & 0 \\
0 & 0 & \kappa_{+}^{+} & \kappa_{-}^{+} & \kappa_{+}^{-} & \kappa_{-}^{-} & -\kappa_{+}^{-} & -\kappa_{-}^{-} \\
0 & 0 & f_{+} \kappa_{+}^{+} & f_{-} \kappa_{-}^{+} & l_{+} \kappa_{+}^{-} & l_{-} \kappa_{-}^{-} & -g_{+} \kappa_{+}^{-} & -g_{-} \kappa_{-}^{-} \\
0 & 0 & \lambda_{+} \kappa_{+}^{-} & \lambda_{-} \kappa_{-}^{+} & -\lambda_{+} \kappa_{+}^{-} & -\lambda_{-} \kappa_{-}^{-} & \lambda_{+} \kappa_{+}^{-} & \lambda_{-} \kappa_{-}^{-} \\
0 & 0 & f_{+} \lambda_{+} \kappa_{+}^{+} & f_{-} \lambda_{-} \kappa_{-}^{+} & -l_{+} \lambda_{+} \kappa_{+}^{-} & -l_{-} \lambda_{-} \kappa_{-}^{-} & g_{+} \lambda_{+} \kappa_{+}^{-} & g_{-} \lambda_{-} \kappa_{-}^{-}
\end{array}\right)\left(\begin{array}{c}
C_{1} \\
C_{2} \\
C_{3} \\
C_{4} \\
C_{5} \\
C_{6} \\
C_{7} \\
C_{8}
\end{array}\right)=0
$$

where, $h_{ \pm}=\left(i \lambda_{ \pm}-k_{y}^{\prime}\right)^{2} /\left(\epsilon+u_{b}\right), l_{ \pm}=\left(i \lambda_{ \pm}+k_{y}^{\prime}\right)^{2} /\left(\epsilon-u_{b}\right)$, $\kappa_{ \pm}^{+}=\exp \left(i \lambda_{ \pm} d\right)$ and $\kappa_{ \pm}^{-}=\exp \left(-i \lambda_{ \pm} d\right)$. Setting the determinant to zero gives the energy spectrum.

\section{SINGLE KINK}

\section{A. Influence of the smoothness of the kink profile}

In the general case of $\delta \neq 0$, we solve the set of secondorder differential Eqs. 3(a) and 3(b) numerically, using the finite-difference technique. Figure 2(a) shows the spectrum for a single potential kink as a function of the wave vector along the kink for zero magnetic field. We consider a relatively sharp kink, i.e., $\delta=1$, and compare the numerical results with the analytical solution (dashed black curves) from Eq. (9) for the case of a sharp profile $(\delta=0)$. The shaded region corresponds to the continuum of free states. The solid red curves correspond to the energy levels of a biased BLG, which can be obtained using Eq. (1) as

$$
\epsilon= \pm \sqrt{k_{y}^{\prime 4}+u_{b}^{2}}
$$

The dotted horizontal lines correspond to $\epsilon= \pm u_{b}= \pm 0.25$ and $\epsilon=0$. These results are valid in the vicinity of a single valley $(K)$ and show that the topological states have a unidirectional character of propagation, i.e., they are chiral states $^{9}$ with positive group velocity. The topological levels can be fitted to $\epsilon=a \sqrt{\left(k_{y}^{\prime}-k_{0}\right)^{4}+\left(u_{b} / a\right)^{2}}-1 / 2$ with $a=0.5$ and $k_{0}=-0.65$ being the fitting parameters (see green solid curve). For localized states around the $K^{\prime}$ valley, we have $E_{K^{\prime}}\left(k_{y}\right)=-E_{K}\left(k_{y}\right)$ and the charge carriers move in the opposite direction. In order to consider the energy levels for the $K^{\prime}$ valley, $u(x)$ in Eqs. (3) should be replaced with $-u(x)$. Then, using the transformations $\epsilon \rightarrow-\epsilon$ and $\varphi_{a} \rightarrow-\varphi_{a}$ (or $\left.\varphi_{b} \rightarrow-\varphi_{b}\right)$ leads to the same equation as for the $K$ valley. Thus, the $E_{K^{\prime}}\left(k_{y}\right)=-E_{K}\left(k_{y}\right)$ symmetry remains even in 


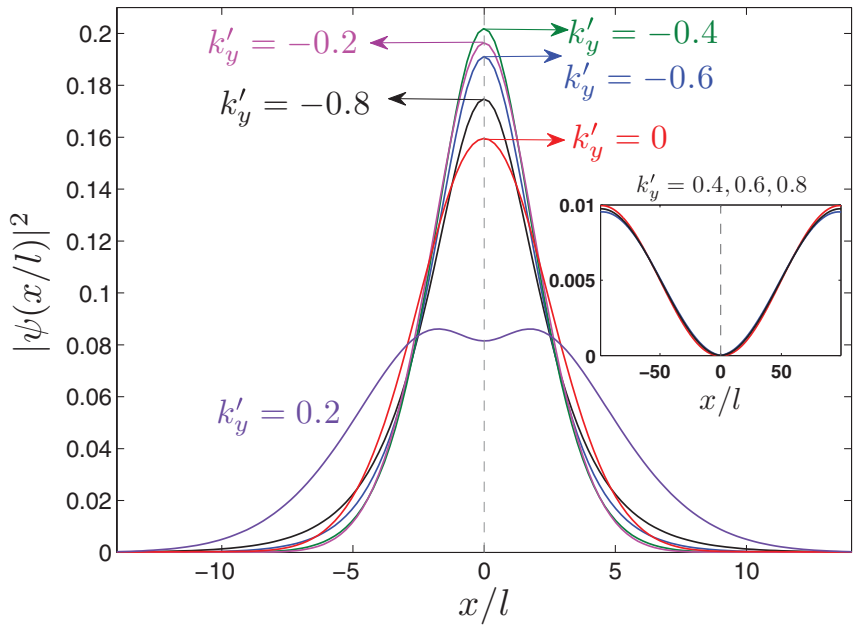

FIG. 3. (Color online) The probability densities of the topological state in $k_{y}^{\prime}=-0.8,-0.6, \ldots$, which correspond to the state indicated by (1) in Fig. (2). The inset shows the probability density for $k_{y}^{\prime}=$ $0.4,0.6$, and 0.8 .

the presence of a uniform perpendicular magnetic field (i.e., $\beta \neq 0$ ). Notice that the wave spinors corresponding to the $K$ and the $K^{\prime}$ valleys are related to each other by $\varphi_{a}^{K} \rightarrow-\varphi_{a}^{K^{\prime}}$ (or $\varphi_{b}^{K} \rightarrow-\varphi_{b}^{K^{\prime}}$ ), while the sign of the other component does not change.

Figures 2(b) and 2(c) present the real parts of the spinor components and the probability density for the states indicated by the arrows $\mathrm{b}$ and a in Fig. 2(a), corresponding to $k_{y}^{\prime}=-0.28$ [Fig. 2(b)] and $k_{y}^{\prime}=0.28$ [Fig. 2(c)]. These electron states are localized at the position of the potential kink. Notice that the solutions of Eqs. (3) are related by the transformations $\varphi_{a} \rightarrow$ $-\varphi_{b}, \varphi_{b} \rightarrow \varphi_{a}, k_{y}^{\prime} \rightarrow-k_{y}^{\prime}$, and $\epsilon \rightarrow-\epsilon$ and, consequently, for $\epsilon=0$, the solutions in Figs. 2(b) and 2(c) have the same probability distribution. For the case of $k_{y}^{\prime}=0$, the solutions of Eq. (8) are $\epsilon_{ \pm}= \pm u_{b} / \sqrt{2}$, which result in the following wave spinors:

$$
\begin{gathered}
\varphi_{a}^{<}=(1 \mp \sqrt{2})\left[e^{-i\left(\lambda_{+} x^{\prime}+\frac{\pi}{4}\right)} \mp e^{i\left(\lambda_{-} x^{\prime}+\frac{\pi}{2}\right)}\right], \\
\varphi_{a}^{>}=e^{i\left(\lambda_{+} x^{\prime}+\frac{\pi}{4}\right)} \mp e^{-i \lambda_{-} x^{\prime}}, \\
\varphi_{b}^{<}=-\frac{(1 \mp \sqrt{2})}{\left(\epsilon_{ \pm}-u_{b}\right)}\left[\lambda_{+}^{2} e^{-i\left(\lambda_{+} x^{\prime}+\frac{\pi}{4}\right)} \mp \lambda_{-}^{2} e^{i\left(\lambda_{-} x^{\prime}+\frac{\pi}{2}\right)}\right], \\
\varphi_{b}^{>}=\frac{-1}{\epsilon_{ \pm}+u_{b}}\left[\lambda_{+}^{2} e^{i\left(\lambda_{+} x^{\prime}+\frac{\pi}{4}\right)} \mp \lambda_{-}^{2} e^{-i \lambda_{-} x^{\prime}}\right],
\end{gathered}
$$

where $\lambda_{ \pm}=\gamma(1 \pm i)$ with $\gamma$ being $\sqrt{u_{b} / 8}$. Notice that, in the above equations, $e^{ \pm i \lambda_{ \pm} x^{\prime}}$ leads to an oscillating contribution $e^{ \pm i \Re\left(\lambda_{ \pm}\right) x^{\prime}}$ with an evanescent $e^{\mp \Im\left(\lambda_{ \pm}\right) x^{\prime}}$ part. The oscillating part is strongly damped and, therefore, Eqs. (13) correspond to localized wave spinors. Expanding Eqs. (13) around $x^{\prime}=0$, we obtain, for the second derivative of the wave spinors,

$$
\begin{gathered}
\frac{\partial^{2}}{\partial x^{\prime 2}} \Re\left[\varphi_{a}^{<}\left(x^{\prime} \rightarrow 0\right)\right]=(1 \pm \sqrt{2})(2+\sqrt{2}) \gamma, \\
\frac{\partial^{2}}{\partial x^{\prime 2}} \Re\left[\varphi_{b}^{<}\left(x^{\prime} \rightarrow 0\right)\right]=\frac{(4-2 \sqrt{2}) \gamma^{3}}{\epsilon_{ \pm}-u_{b}}<0 .
\end{gathered}
$$

This indicates that $\Re\left[\varphi_{a}\right]\left(\Re\left[\varphi_{b}\right]\right)$ has its maximum value located at $x^{\prime}<0\left(x^{\prime}>0\right)$ for $\epsilon_{+}=u_{b} / \sqrt{2}$, while the opposite is found for $\epsilon_{-}=-u_{b} / \sqrt{2}$, which is also evident from Figs. 2(b) and 2(c).

In Fig. 3, we show the probability densities corresponding to one of the topological branches [for the state that is labeled by (1) in Fig. 2(a)] at several $k_{y}^{\prime}$ values. As shown in the inset of Fig. 3 for those $k_{y}^{\prime}$ values where the topological state merged with the continuum spectrum, the carries are no longer confined by the kink potential.
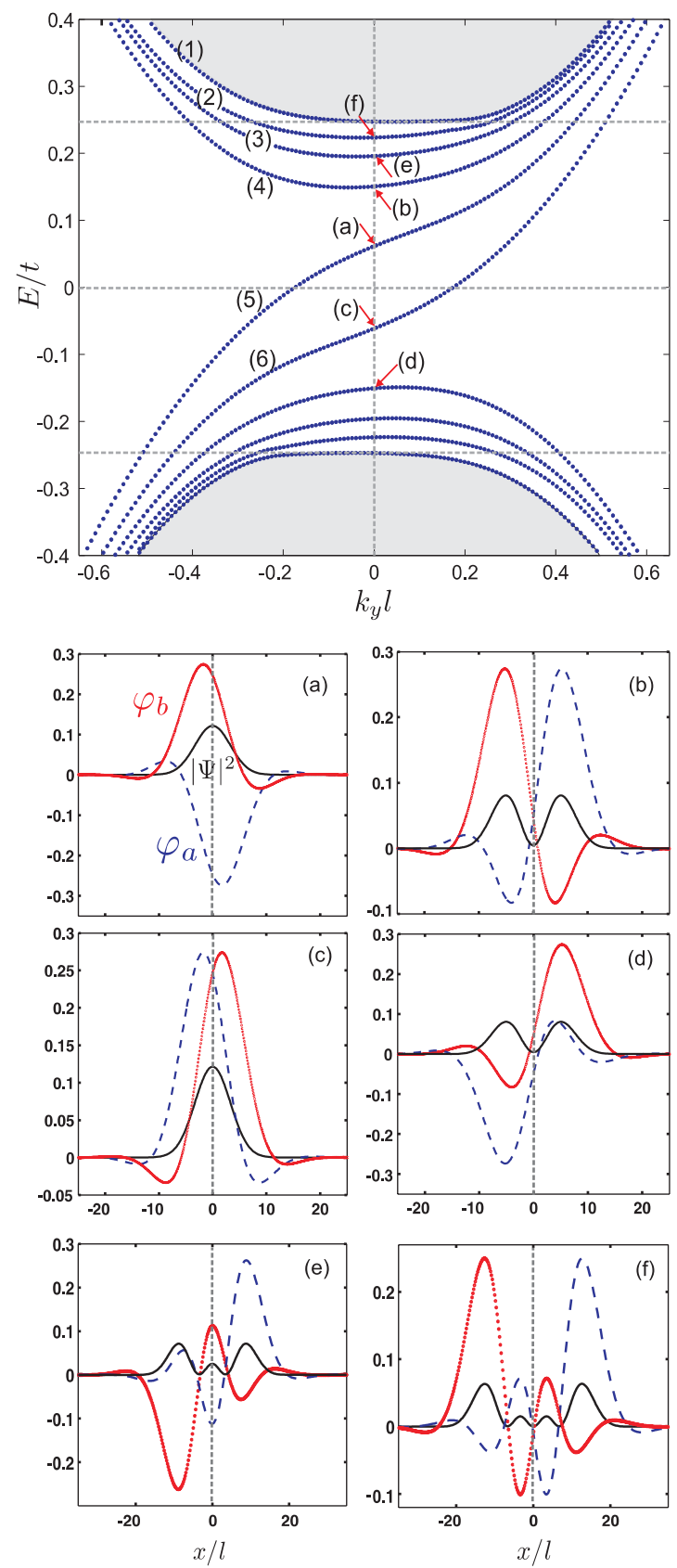

FIG. 4. (Color online) Upper panel: Energy levels for a single kink profile in bilayer graphene with $u_{b}=0.25$ and $\delta=10$. The energy states indicated by (a) and (c) are chiral states and those indicated by (b), (d), (e), and (f) are the extra-bound states. Lower panels: Real parts of the wave spinors and the corresponding probability density for the two first electron and hole energy levels at $k_{y}^{\prime}=0$ as indicated in the upper panel. 
(a) Sharp kink
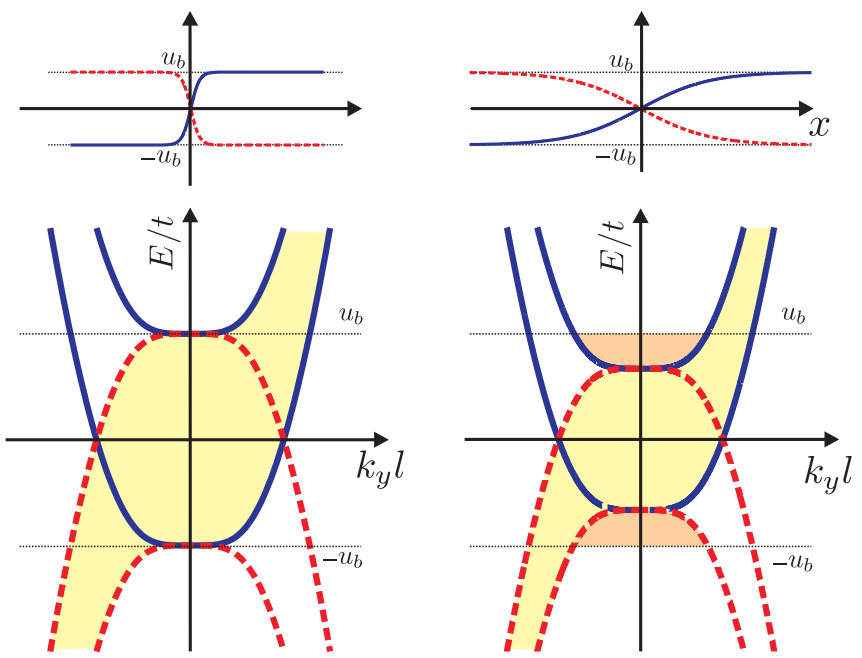

FIG. 5. (Color online) Upper panels: applied potential profile $u(x)$ to the upper (solid blue curve) and the lower layer (red dashed curve) for (a) sharp and (b) smooth kink profiles. Lower panel: schematic representation of the energy spectrum in $\left(E, k_{y}\right)$ space, corresponding to the solid blue and red dashed potential profiles near the kink region. Topological states can be found in the yellow region. For the smooth profile (b), extra bound states can be found in the orange region.

Next, we increase the smoothness of the kink potential and investigate how the energy spectrum changes. In Fig. 4, the energy levels as a function of $k_{y}$ are shown for the smooth kink profile $\delta=10$, where, in addition to the chiral states, several branches are seen that are split off from the continuum. In order to understand the physical origin of those new states, we show in the lower panels of Fig. 5 a cartoon of the low-energy spectra for the (a) sharp and (b) smooth profiles where the chiral states appear in the yellow regions and those additional states are found in the orange region. Increasing the smoothness of the kink potential leads to the creation of a region below the energy gap, which allows for carriers to be confined near the kink. Therefore, extra bound states can be created in the orange region [lower panel in Fig. 5(b)]. The wave functions for $k_{y}=0$ of the two chiral states and the new bound states are shown in the lower panels of Fig. 4. The new bound states are also bound in the $x$ direction near $x=0$, but the electron states are more extended and have a clear nodal character near $x=0$.

Figure 6 shows the velocity of the carriers for the states that are indicated by (1),(2), . . in Fig. 4 . The chiral states $[(5),(6)]$ are only shown for the $K$ valley and they have positive velocity. The curves (5), (6) can be fitted to $v / v_{F} \approx a \sqrt{\left(k_{y}^{\prime}-k_{0}^{\prime}\right)^{2}+b^{2}}$ (see the solid gray curves) with $a=1.8, b=0.15$ being the fitting parameters and $k_{0}^{\prime}= \pm 0.08$ corresponds to the minimum point in the curves (5) and (6). Notice that the extra bound states $[(2),(3),(4)]$ have a slightly nonzero velocity at $k_{y}^{\prime}=0$, which is a consequence of the asymmetric energy dispersion as seen in Fig. 4. Curve (1) corresponds to the energy spectrum of a biased BLG, which is given by Eq. (12) and results in the velocity $v / v_{F}=\left[\partial \epsilon / \partial k_{y}^{\prime}\right]=2 k_{y}^{\prime 3} / \sqrt{k_{y}^{\prime 4}+u_{b}^{2}}$, which is zero for $k_{y}^{\prime}=0$ in a biased BLG (black solid curve in Fig. 6).

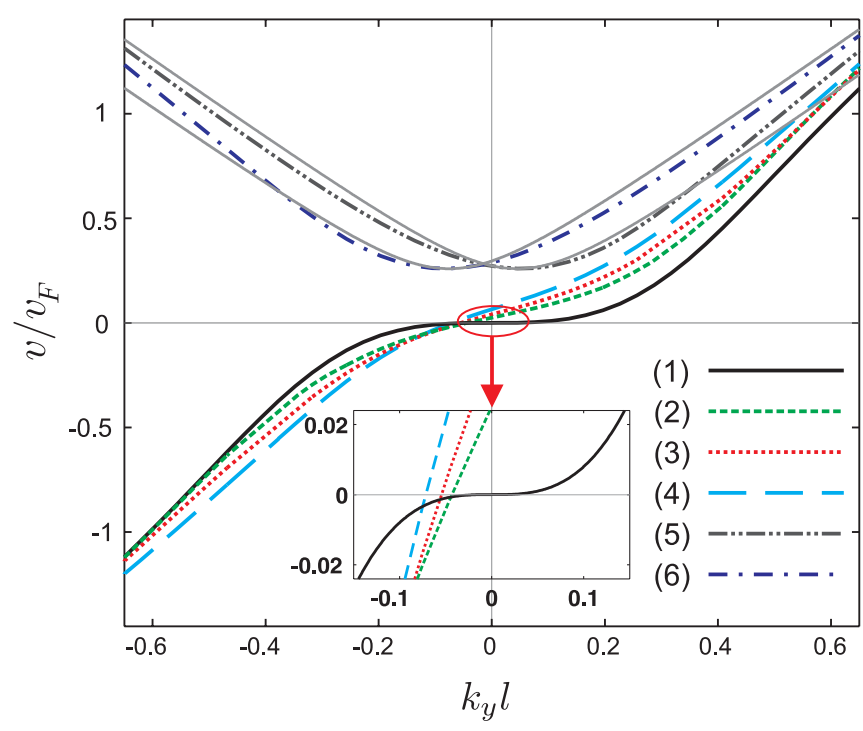

FIG. 6. (Color online) The carrier velocity in the single kink profile for the energy levels, which are indicated by (1),(2), . . in Fig. 4. The gray solid curves are the fitted functions to curves (5) and (6).

As mentioned before, for smooth kink potentials, additional $1 \mathrm{D}$ bound states appear and the number of these bound states can be related to the height of the gate voltage $u_{b}$ and the smoothness $(\delta)$ at the interface. Figure 7 shows the number of these extra bound states for three different $u_{b}$ values as function of the width $\delta$. The first bound state for $u_{b}=0.1,0.25,0.5$ appears, respectively, at $\delta \approx 6,4,2$ in the absence of magnetic field. Notice also that, for fixed $\delta$, the number of extra bound states increases with $u_{b}$ in agreement with the qualitative picture shown in Fig. 5(b).

We also calculate the transmission of an electron through the kink structure in a system of size $-L_{x} / 2<x<L_{x} / 2$ and $-L_{y} / 2<y<L_{y} / 2$. No bias nor magnetic field is assumed in the $x<-L_{x} / 2$ and $x>L_{x} / 2$ regions. We assume that $L_{y} \gg$ $L_{x}$ and the electrons are free to move in the $y$ direction, whereas they are confined in the $x$ direction. Associated with each real $\lambda_{ \pm}$, there are two right (left) propagating modes $\psi_{ \pm}^{>}\left(\psi_{ \pm}^{<}\right)$, which are given by Eqs. (7). In the region I $\left(x<-L_{x} / 2\right)$, two

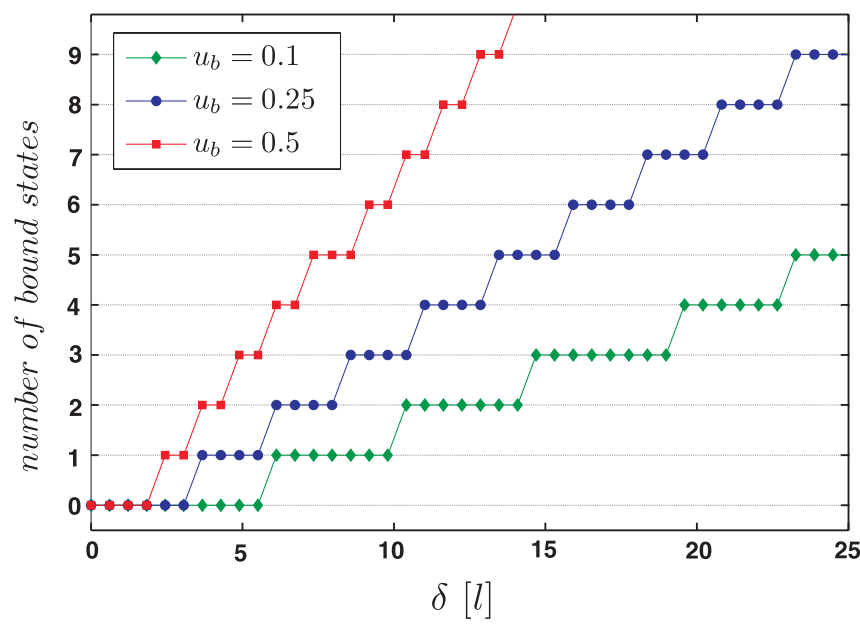

FIG. 7. (Color online) Number of additional bound states as a function of the width of the interface $\delta$ for $u_{b}=0.1,0.25$, and 0.5 . 

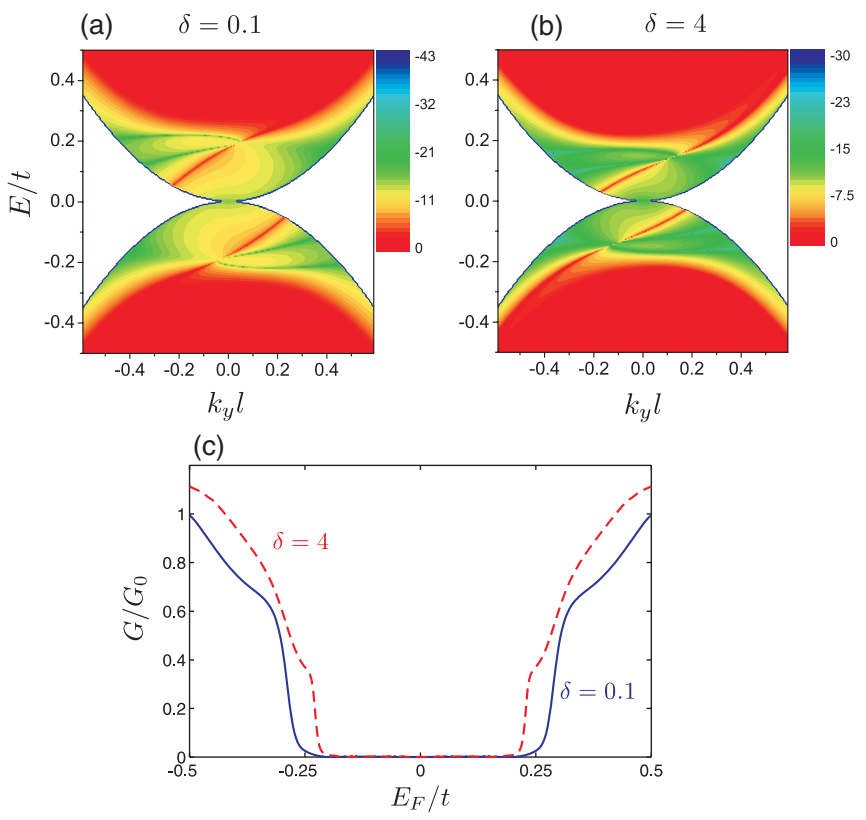

FIG. 8. (Color online) (a), (b) Contour plot of the transmission $T$ (in logarithmic scale) through a single kink structure with $L_{x}=20$ $(\approx 32 \mathrm{~nm}), u_{b}=0.25$ for (a) $\delta=0.1$ and (b) $\delta=4$. (c) Conductance $G / G_{0}$ vs Fermi energy of a single kink profile for the same parameters as (a), (b).

incident right-traveling modes $\psi_{ \pm}^{>}$can be reflected into two left-traveling modes $\psi_{ \pm}^{<}$

$$
\Psi_{ \pm}^{\mathrm{I}}=\psi_{ \pm}^{>}+r_{ \pm}^{+} \psi_{+}^{<}+r_{ \pm}^{-} \psi_{-}^{<},
$$

where $t_{ \pm}^{ \pm}\left(r_{ \pm}^{ \pm}\right)$are the transmission (reflection) amplitudes. The propagating modes in region I can also be transmitted to region III $\left(x>L_{x} / 2\right)$ in the right-traveling modes

$$
\Psi_{ \pm}^{\mathrm{III}}=t_{ \pm}^{+} \psi_{+}^{>}+t_{ \pm}^{-} \psi_{-}^{>} .
$$

The wave functions in regions I and III can be connected by the transfer matrix $M$, where at the kink-potential boundaries we have

$$
\Psi_{ \pm}^{\mathrm{I}}\left(-L_{x} / 2\right)=M \Psi_{ \pm}^{\mathrm{III}}\left(L_{x} / 2\right) .
$$

The transmission (or reflection) amplitude can be found by substituting Eqs. (15) and (16) in the above equation. The four transmission amplitudes $t_{ \pm}$for given $\epsilon$ and $k_{y}$ can be combined in the transmission matrix

$$
t\left(\epsilon, k_{y}\right)=\left(\begin{array}{ll}
t_{+}^{+} & t_{-}^{+} \\
t_{+}^{-} & t_{-}^{-}
\end{array}\right) .
$$

The total transmission amplitude $T$ is given by ${ }^{15} T=\operatorname{Tr}\left(t t^{\dagger}\right)$. The two-terminal conductance of such an asymmetric potential profile in bilayer graphene can be calculated using the Landauer formula, which is given by ${ }^{16,17}$

$$
G=G_{0} \int T\left(E_{F}, k_{y}^{\prime}\right) d k_{y}^{\prime} .
$$

Here, $G_{0}=\left(2 e^{2} / h\right)\left(L_{y}\left|E_{F}\right| / \pi \hbar v_{F}\right)$ is the conductance unit per valley and per spin. In Figs. 8(a) and 8(b), we show a contour plot of the transmission probability in logarithmic scale for the kink structure with $L_{x}=20$ (in dimensionless unit). The transmission probability has the symmetry $T\left(k_{y}, E\right)=T\left(-k_{y},-E\right)$. The conductance as a function of the Fermi energy for the single kink profile is shown in Fig. 8(c) for $\delta=0.1$ (blue solid curve) and $\delta=4$ (red dashed curve). For the case $\delta=4$, the smoothness of the potential at $x=0$ leads to a higher transmittance and consequently a higher conductance around $\epsilon \approx u_{b}$ [see Fig. 8(b) and the dashed curve in Fig. 8(c)].

\section{B. Magnetic field dependence}

Dependence of the energies of the 1D bound states on an external magnetic field is shown in Fig. 9 for (a) $k_{y}^{\prime}=0$ and (b) $k_{y}^{\prime}=0.15$. In order to show the effect of a magnetic field on the chiral states (blue solid curves) and the other localized bound states (red dashed curves), we present the results for a smooth potential (i.e., $\delta=10$ ). It is seen that the chiral states are very weakly influenced by the magnetic field. This is a consequence of the strong confinement of these states in the kink potential [see Figs. 3 and Figs. 4(a) and 4(c)]. In a semiclassical view, the movement of the carriers is constrained by the kink potential and that, together with the unidirectional propagation, prevents the formation of cyclotron orbits. For the energy levels above the chiral states, the energy values increase as the magnetic field increases because of the weaker confinement of these states as is apparent from Figs. 4(b), 4(d), 4(e), and 4(f).

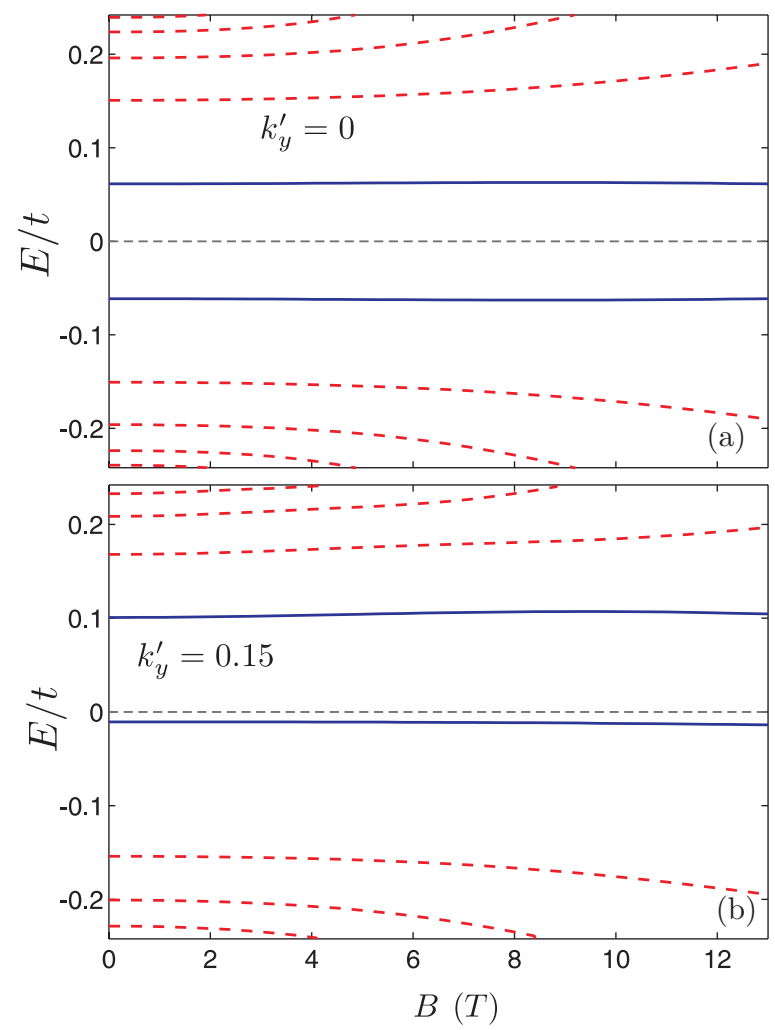

FIG. 9. (Color online) Energy levels of a single potential kink profile as a function of the external magnetic field with $u_{b}=0.25$ and $\delta=10$ for (a) $k_{y} l=0$ and (b) $k_{y} l=0.15$. The full blue curves are the topological states and the dashed red curves are the extra bound states. 
(a)
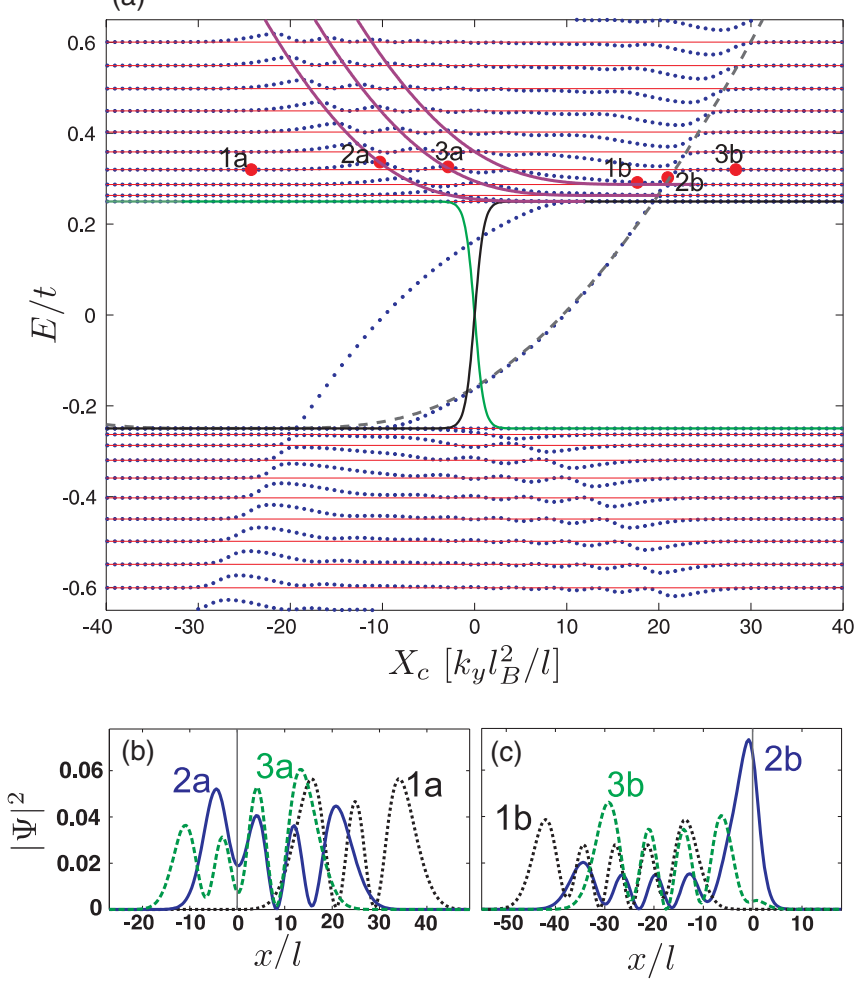

FIG. 10. (Color online) (a) Energy spectrum of a single kink profile in bilayer graphene as a function of the cyclotron orbit coordinate $X_{c}$ for $B=7 \mathrm{~T}, u_{b}=0.25$, and $\delta=1$. The dashed gray curve shows a fitted function to the numerical results. The solid black and green curves are, respectively, the potential in the upper and lower layers. Solid red lines correspond to the Landau levels of a biased BLG. The solid purple curves show fitted functions (given in the text) to the position of the resonances. (b), (c) The probability densities for the indicated points are shown by red full circles in the spectrum.

Figure 10 shows the spectrum of a sharp $(\delta=1)$ single kink potential in the presence of an external magnetic field $B=7 \mathrm{~T}$ as a function of the orbit center $X_{c}=k_{y} l_{B}^{2} / l$ where $l_{B}=\sqrt{\hbar / e B}$ is the magnetic length. The solid lines represents the applied kink potential to upper (black) and lower (green) layers. The results show that the topological states are practically not affected by the magnetic field. The free-energy region (i.e., $|\epsilon|>u_{b}$ ) in the absence of magnetic field now is replaced with Landau levels (the solid red lines are the Landau levels of a biased bilayer graphene). In some region, the Landau levels are influenced by the kink potential and anticrossings appear in the low-energy spectrum. Some of these anticrossings are situated along the extension of the topological states into the $|\epsilon|>u_{b}$ region. In addition to these anticrossings, the Landau levels display some resonances along the energy levels of a biased BLG [red solid curves in Fig. 2(a)], which can be linked to the edge effects of the potential profile. The position of the resonances can be fitted to $\epsilon=a \sqrt{\left(X_{c}-X_{0}\right)^{4}+\left(\epsilon_{n} / a\right)^{2}}$, where $a=0.0006$ and $X_{0}=$ 9.25 are fitting parameters and $\epsilon_{n}$ indicates the $n$th Landau level of a biased BLG (see solid purple curves). Also, the topological levels can be fitted to $\epsilon=a \sqrt{\left(X_{c}-X_{0}\right)^{4}+\left(u_{b} / a\right)^{2}}$ (dashed gray curve) with $a=0.0003$ and $X_{0}=-26$. Figures 10(b) and $10(\mathrm{c})$ show the probability densities for the points that are

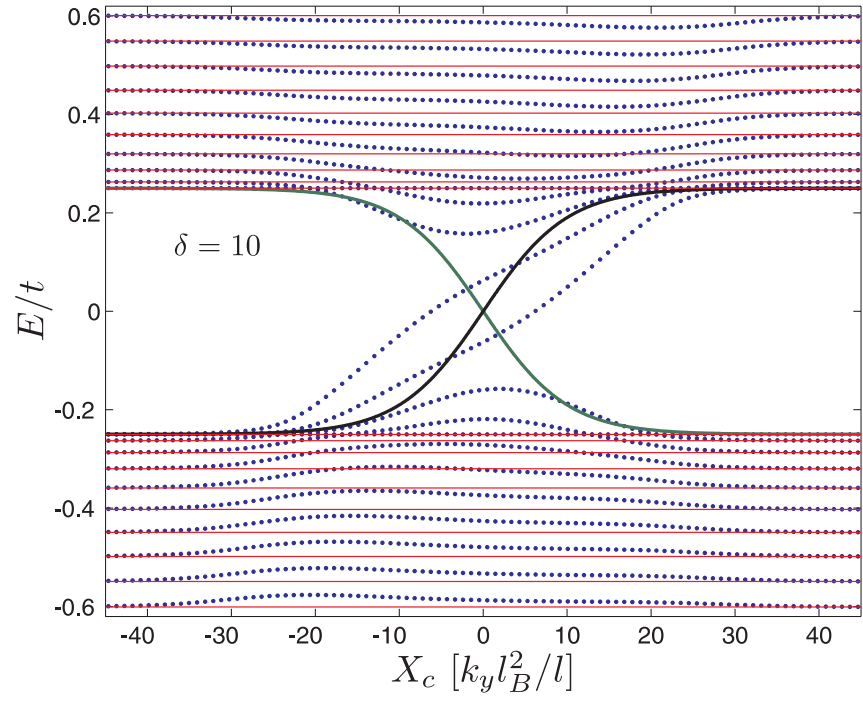

FIG. 11. (Color online) The same as Fig. 10 but for $\delta=10$. The solid black and green curves are the potential, respectively, in the upper and lower layers.

indicated by full red circles in the energy spectrum. For the points on the purple solid curves $(2 \mathrm{a}, 3 \mathrm{a})$, the distribution of the carriers by the magnetic field is influenced by the small confinement by the interface potential [see solid and dashed curves in Fig. 10(b)]. The probability density for the point on the fitted curve along the topological level (2b) shows a higher peak at the kink interface $(x=0)$ indicating that the kink potential acts as an attractive potential [solid curve in Fig. 10(c)]. The other probabilities are clearly those of free electron LL. The result for a smooth kink potential $\delta=10$ is shown in Fig. 11, where the energy values of the extra bound states are increased by the magnetic field and the topological levels are practically not affected by the magnetic field.

The localization of the states is reflected in the position dependence of the current. The current in the $y$ direction is obtained using

$j_{y}=i v_{F}\left[\Psi^{\dagger}\left(\partial_{x} \sigma_{y}-\partial_{y} \sigma_{x}\right) \Psi+\Psi^{T}\left(\partial_{x} \sigma_{y}+\partial_{y} \sigma_{x}\right) \Psi^{*}\right]$,

where $\Psi=\left[\psi_{a}(x, y), \psi_{b}(x, y)\right]^{T}$. By substituting $\Psi(x, y)=$ $e^{i k_{y} y}\left[\varphi_{a}(x), \varphi_{b}(x)\right]^{T}$, we have

$$
j_{y}=2 v_{F}\left[\operatorname{Re}\left\{\varphi_{a}^{*} \partial_{x} \varphi_{b}-\varphi_{b}^{*} \partial_{x} \varphi_{a}\right\}+2 k_{y} \operatorname{Re}\left\{\varphi_{a}^{*} \varphi_{b}\right\}\right] .
$$

The $x$ component of the current vanishes for the confined states. In Fig. 12, the $y$ component of the persistent current for a sharp (blue curves) and smooth (black curves) single potential kink profile is shown as function of the $x$ direction without magnetic field (solid curves) and in the presence of the magnetic field (dashed curves). In the absence of a magnetic field, the current is localized around $x=0$ for both sharp $(\delta=$ $1)$ and smooth $(\delta=10)$ potentials. For a smooth profile, the wave function of the topological states and, consequently, also the current density profile, is broadened [compare Figs. 2(b) and 2(c) with Figs. 4(a) and 4(c)]. A magnetic field shifts the density profile slightly to the right (see the inset of Fig. 12) due to the Lorentz force, and there is also a very small narrowing of the current distribution. 


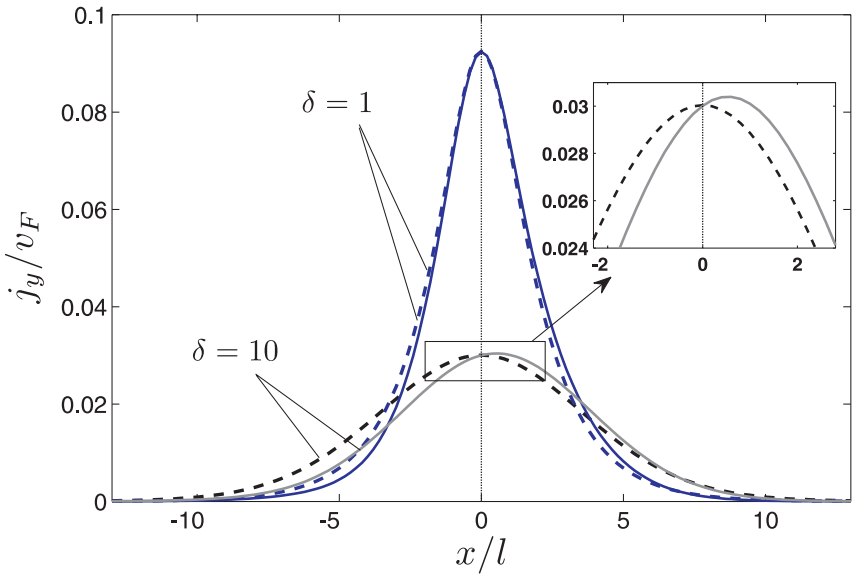

FIG. 12. (Color online) $y$ component of the persistent current corresponding to the topological state of a single kink potential as a function of the $x$ direction for zero magnetic field (dashed curves) and $B=5 \mathrm{~T}$ (solid curves) with $k_{y}^{\prime}=0$ and $u_{b}=0.25$. Blue curves display the current density for $\delta=1$ and black curves are the corresponding results for $\delta=10$.

Next, we consider the density of states (DOS) for the kink potential. The number of $k$ states per unit energy is given by

$$
D(E)=\frac{D_{0}}{2 \pi} \sum_{n} \int d k_{y}^{\prime} \delta\left(\epsilon-\epsilon_{n, k_{y}^{\prime}}\right),
$$

where $D_{0}=\left(\hbar v_{F}\right)^{-1}$. To calculate the DOS numerically, we introduce a Gaussian broadening

$$
\delta\left(\epsilon-\epsilon_{n, k_{y}^{\prime}}\right) \rightarrow \frac{1}{\Gamma \sqrt{\pi}} \exp \left[-\frac{\left(\epsilon-\epsilon_{n, k_{y}^{\prime}}\right)^{2}}{\Gamma^{2}}\right],
$$

where $\Gamma$ is the broadening that is taken as $\Gamma=0.005$ in our calculations. Figure 13 shows the DOS as a function of Fermi energy $E_{F}$ in the absence and presence of an external magnetic field for sharp $(\delta=1)$ and smooth $(\delta=10)$ kink potentials. For a sharp profile, the topological levels contribute an almost constant value to the DOS for $|\epsilon|<u_{b}$ even in the presence

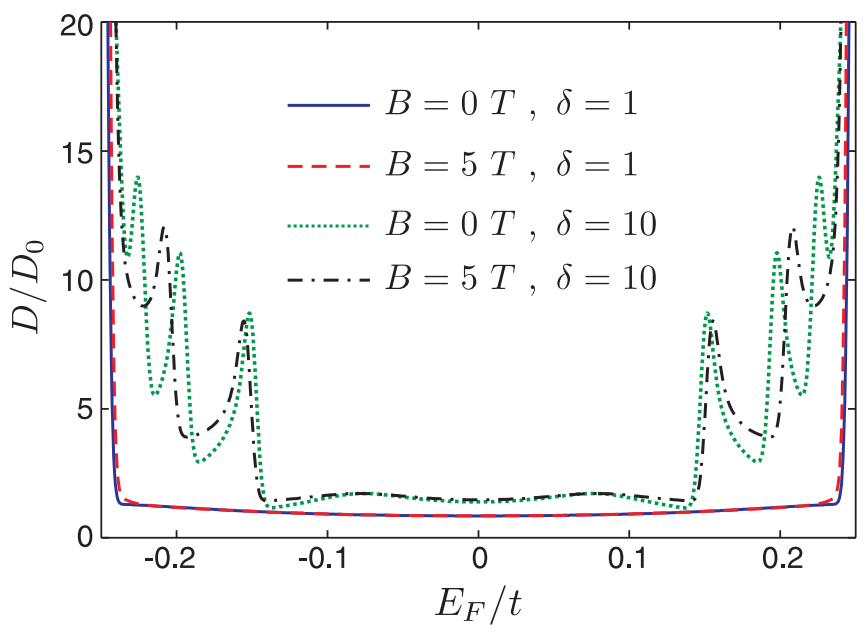

FIG. 13. (Color online) Density of states (DOS) of a single kink potential for $\delta=1$ (solid and dashed curves) and $\delta=10$ (dotted and dashed-dotted curves) with $u_{b}=0.25$ for two different magnetic field values.

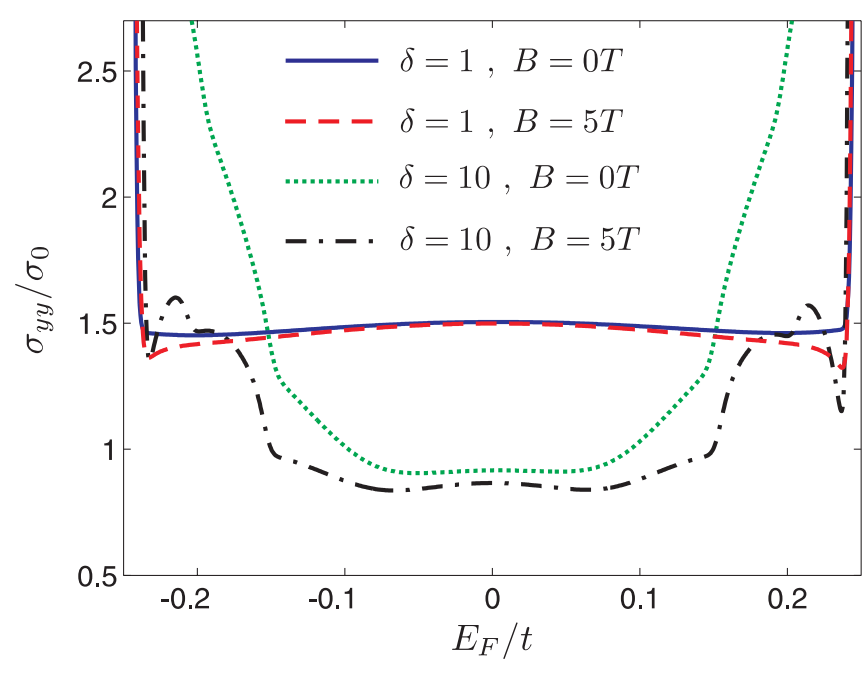

FIG. 14. (Color online) Conductivity $\sigma_{y y}$ as a function of the Fermi energy $E_{F}$ for a single kink potential with $u_{b}=0.25$. Other parameters are indicated in the figure.

of an external magnetic field. For the smooth profile, peaks corresponding to the nontopological levels appear in the DOS and note that only these peaks are shifted in the presence of a magnetic field, while the DOS of the topological states is not affected by the magnetic field.

We now turn to the transport properties of a kink potential and look at the influence of the topological states on the conductivity in the $y$ direction $\left(\sigma_{y y}\right)$. For elastic scattering, the diffusive conductivity $\sigma_{y y}$ is given by ${ }^{18}$

$$
\sigma_{y y}=\frac{e^{2} v_{F}}{2 \pi \hbar k_{B} T} \sum_{n} \int d k_{y}^{\prime} \tau v_{n, y}^{2} f_{n, k_{y}^{\prime}}\left(1-f_{n, k_{y}^{\prime}}\right) .
$$

Here, $T$ is the temperature, $v_{n, y}=\partial \epsilon_{n} / \partial k_{y}^{\prime}$ is the electron velocity, $f_{n, k}=1 /\left\{\exp \left[\left(E_{F}-E_{n, k_{y}^{\prime}}\right) / K_{B} T\right]+1\right\}$ is the equilibrium Fermi-Dirac distribution function, and $\tau$ is the momentum relaxation time. For low temperatures, we assume that $\tau$ is approximately constant, evaluated at the Fermi level $\left(\tau \approx \tau_{F}\right)$, and replace the product $f_{n, k_{y}^{\prime}}\left(1-f_{n, k_{y}^{\prime}}\right) / k_{B} T$ by the delta function given in Eq. (23). The results are presented as a function of $E_{F}$ in Fig. 14 in the units of $\sigma_{0}=e^{2} v_{F} \tau_{F} / \hbar L_{y}$ for both sharp $(\delta=1)$ and smooth $(\delta=10)$ potentials with $B=0$ and $5 \mathrm{~T}$. Due to the robust confinement of the topological levels, the conductivity is constant in the energy gap even for a nonzero magnetic field (solid blue curve for $B=0 \mathrm{~T}$ and red dashed curve for $B=5 \mathrm{~T}$ ). The extra localized levels in the case of $\delta=10$ lead to an increasing conductivity as a function of $E_{F}$. Note that, in the presence of an external magnetic field, some of the additional electron (hole) states are shifted up (down) in energy (see Figs. 4 and 11), which results in smaller $\sigma_{y y}$ at the $\left|\epsilon_{F}\right|<u_{b}$ region compared to the conductivity in the absence of magnetic field (black dotted-dashed curve).

\section{KINK-ANTIKINK}

\section{A. Zero magnetic field}

Next, we consider a potential profile with a pair kinkantikink. The kink-antikink potential is modeled by

$$
u\left(x^{\prime}\right)=u_{b}\left[\tanh \left(\frac{x^{\prime}-d}{\delta}\right)-\tanh \left(\frac{x^{\prime}+d}{\delta}\right)+1\right] \text {, }
$$



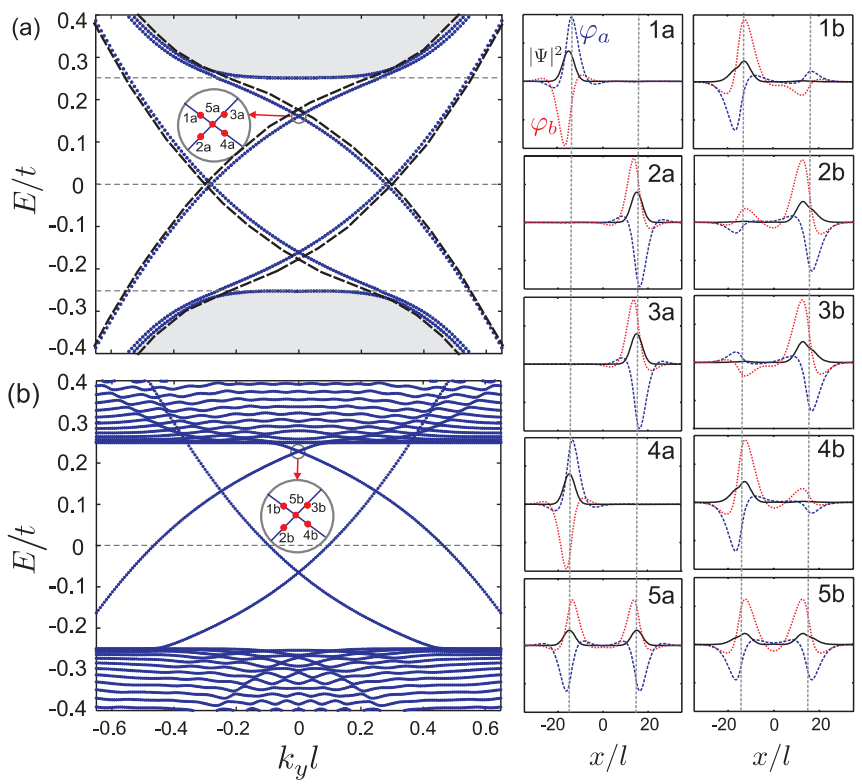

FIG. 15. (Color online) Left panels: Energy levels of a kinkantikink profile in bilayer graphene with $u_{b}=0.25, \delta=1, d=15$ for (a) $B=0 \mathrm{~T}$ and (b) $B=3 \mathrm{~T}$. The black dashed curves display analytical results as obtained from Eq. (11). Right panels: Real part of the wave spinors and the corresponding probability density for the points that are indicated in panels (a) and (b).

where $d$ is the distance between the kink and the antikink in units of $l$. The spectrum of the localized states in the absence of a magnetic field is shown in Fig. 15(a) for $u_{b}=0.25, \delta=1$, and $d=15$. The black dashed curves are the analytical results for $\delta=0$, which are obtained using Eq. (11). Note that there are only two chiral states per kink, which leads to the appearance of crossing points in the energy spectrum (at $E=0$ and $k_{y}^{\prime}=0$ ). The spinor components and probability densities associated with the points indicated inside the circle in Fig. 15(a) are shown in the panels $(1 \mathrm{a}, 2 \mathrm{a}, \ldots, 5 \mathrm{a})$. In the absence of a magnetic field and for
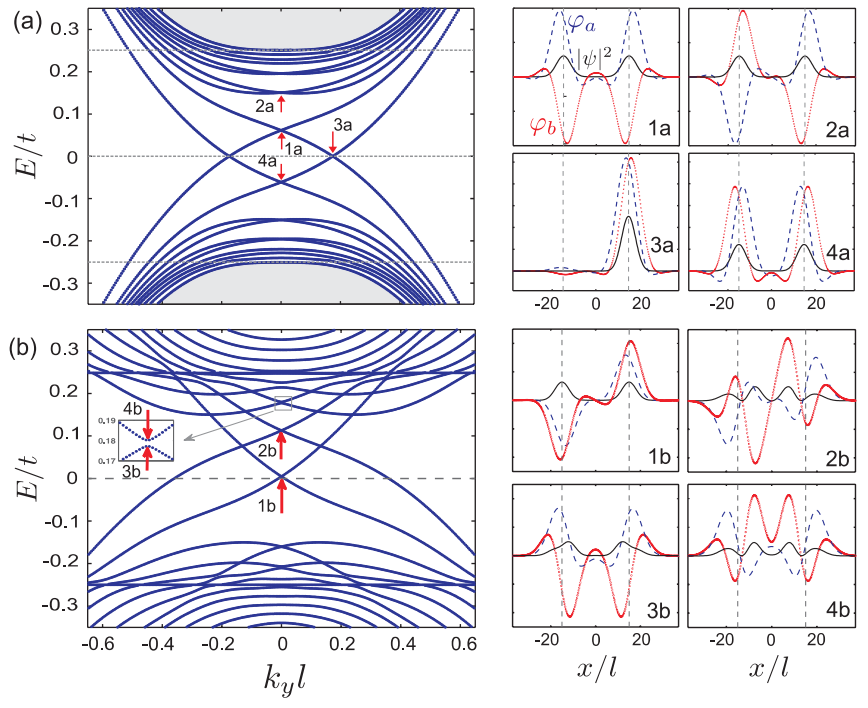

FIG. 16. (Color online) The same as Fig. 15 but for a smooth kink-antikink profile with $\delta=10$. the points around the energy level crossing, the carriers are strongly confined at either the position of the kink or antikink. The wave function corresponding to an energy at the crossing point (5a) is localized at both the kink and antikink.

Next, we investigate smooth potential kink profiles. In Fig. 16(a), the energy spectrum of a smooth kink-antikink profile (i.e., $\delta=10$ ) is presented for zero magnetic field. As in the case of the single kink profile, additional bound states appear in the energy spectrum. The overlap between theses states leads to the appearance of crossing points in the energy spectrum. The wave spinors and the corresponding probability density for the points indicated by arrows in Fig. 16(a) are shown in panels (1a, 2a, 3a, 4a). In the absence of a magnetic field and for $k_{y}^{\prime}=0$, the states are localized at both kink and antikink (panels 1a, 2a, and 4a), whereas panel 3a shows that the confinement tends to the kink or antikink at $k_{y}^{\prime} \neq 0$.

Decreasing the distance between the kink and antikink generates an unperfect kink-antikink profile. ${ }^{19}$ This profile is illustrated in Fig. 17(a). The energy spectrum of such a profile is shown in Fig. 17(b) for $B=0 \mathrm{~T}, \delta=1$, and $d=3(\approx 5 \mathrm{~nm})$. The analytical results [obtained from Eq. (11)] for $\delta=0$ are shown by the black dashed curves. Now, the crossing points in the energy spectrum for the case of $d=15$ [see Fig. 15(a)] are replaced with anticrossings and an energy gap $E_{g}$ appears in the energy spectrum. The positions of these minigaps move

(a)
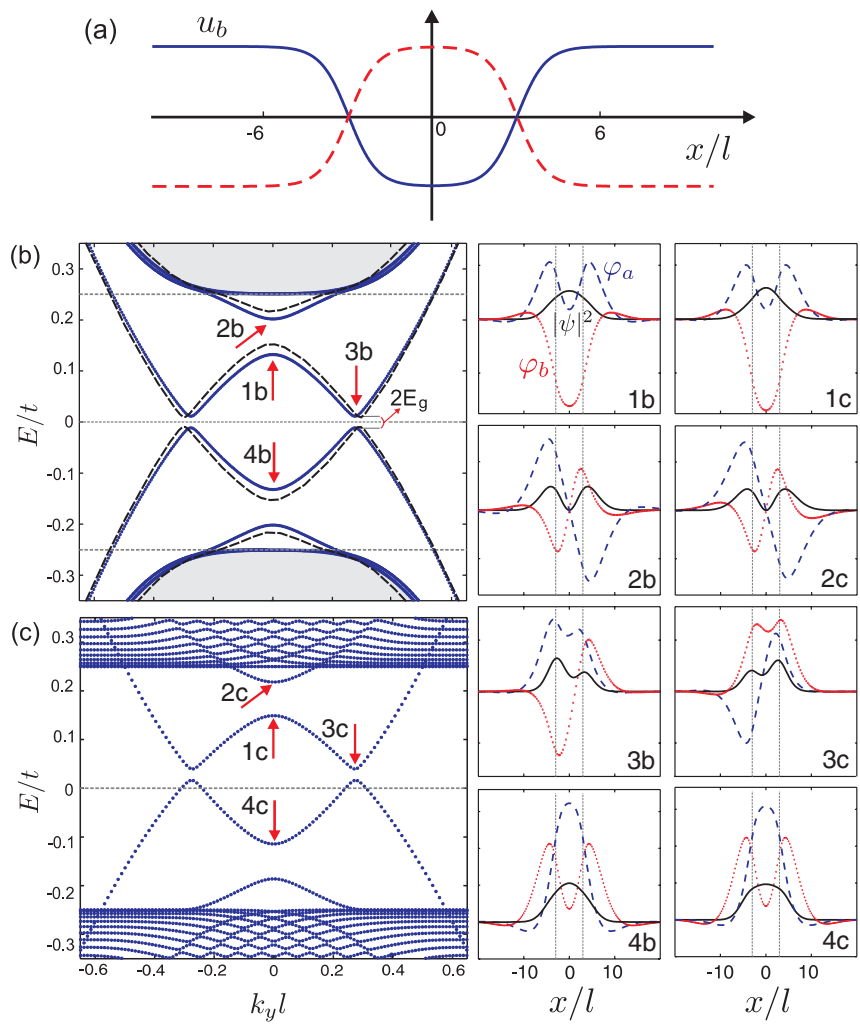

FIG. 17. (Color online) Energy levels of a sharp kink-antikink profile with $u_{b}=0.25, d=3(\approx 5 \mathrm{~nm})$, and $\delta=1$ [the profile is depicted in (a)] for (b) $B=0 \mathrm{~T}$ and (c) $B=3 \mathrm{~T}$. The black dashed curves in panel (b) display analytical results as obtained from Eq. (11). Right panels show the real part of the wave spinors and the corresponding probability density for the points at the energy spectrum that are indicated by arrows in panels (b) and (c). 


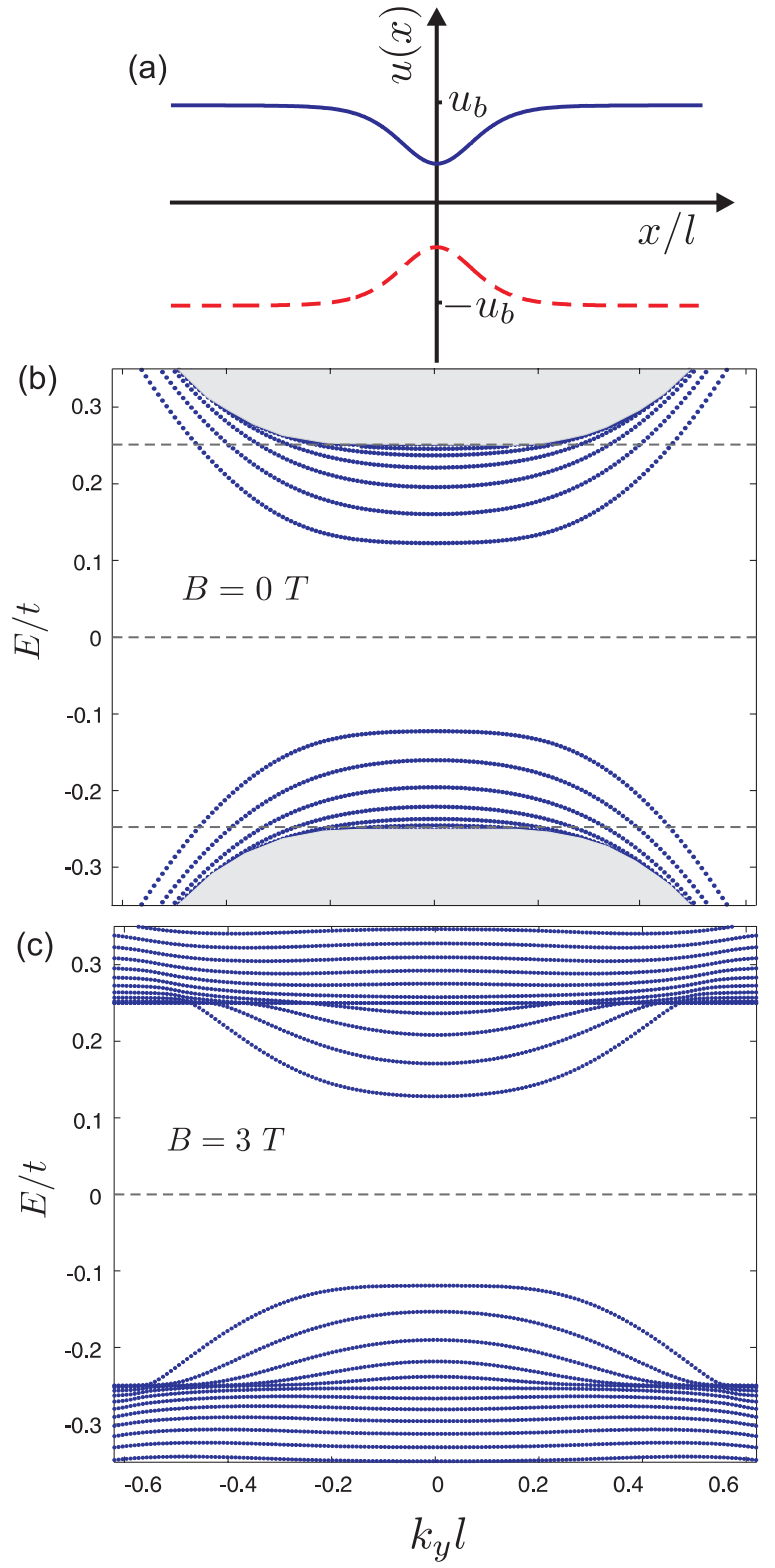

FIG. 18. (Color online) Energy levels of a smooth kink-antikink profile on bilayer graphene with $u_{b}=0.25, d=3$, and $\delta=10$ for (a) $B=0 \mathrm{~T}$ and (b) $B=3 \mathrm{~T}$.

when we increase the magnetic field as is apparent from Fig. 17(c). The panels $(1 b, 2 b, 3 b, 4 b)$ show the real parts of the wave spinors and corresponding probability density for the indicated points in Fig. 17(b) by red arrows. Note that, due to the decreasing distance of the kink and antikink, the carriers can be localized between the kink and antikink.

Figure 18(a) displays the energy spectrum of a smooth $(\delta=10)$ kink-antikink potential with $d=3$ for $B=0 \mathrm{~T}$. Now, the kink and antikink are close to each other and the smoothness of the potential leads to extra localized levels. Therefore, the crossing and anticrossing points between the additional bound states are seen to disappear, and the energy gap between the topological levels is increased. The magnitude of the energy gap $E_{g}$ depends on the width of the interface region $\delta$, the maximum value of the potential $u_{b}$, and the distance between the kink and antikink. This is shown in
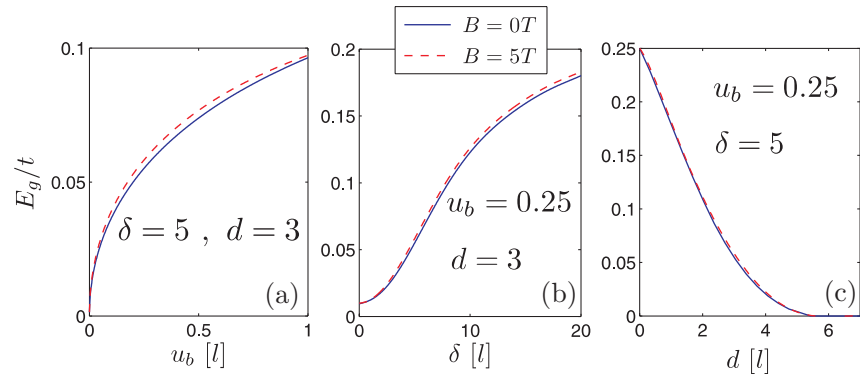

FIG. 19. (Color online) The energy gap $E_{g}$ [indicated in Fig. 17(b)] as a function of (a) maximum amplitude of the gate voltage $u_{b}$, (b) the width of kink profile $\delta$, and (c) the position of the kink and antikink $d$. Other parameters are shown in the figures.

Fig. 19 , where $E_{g}$ is plotted as a function of $u_{b}, \delta$, and $d$, respectively, in panels (a), (b), and (c) in the absence of magnetic field (blue solid curves). As shown in Figs. 19(a) and 19(b), the energy gap is an increasing function of $u_{b}$ and $\delta$. When $\delta$ increases, the first energy level at the spectrum changes from a Mexican hat shape to a parabola. Therefore, $E_{g}$ increases with increasing $\delta$ [compare the potentials illustrated in Figs. 17(a) and 18(a)]. Increasing the distance of the kink and antikink results in perfect unidirectional states, and the gap disappears [Fig. 19(c)].

Next, we consider the transmittance of a kink-antikink potential. In Fig. 20, we show a contour plot of the transmission probability (in logarithmic scale) for the kink-antikink structure with $L_{x}=24$ for (a) $\delta=0.1$ (sharp) and (b) $\delta=4$ (smooth) potentials. The results show a nonzero region for the transmittance below the gap where the topological levels corresponding to the kink and antikink cross each other. The conductance as a function of Fermi energy is plotted in
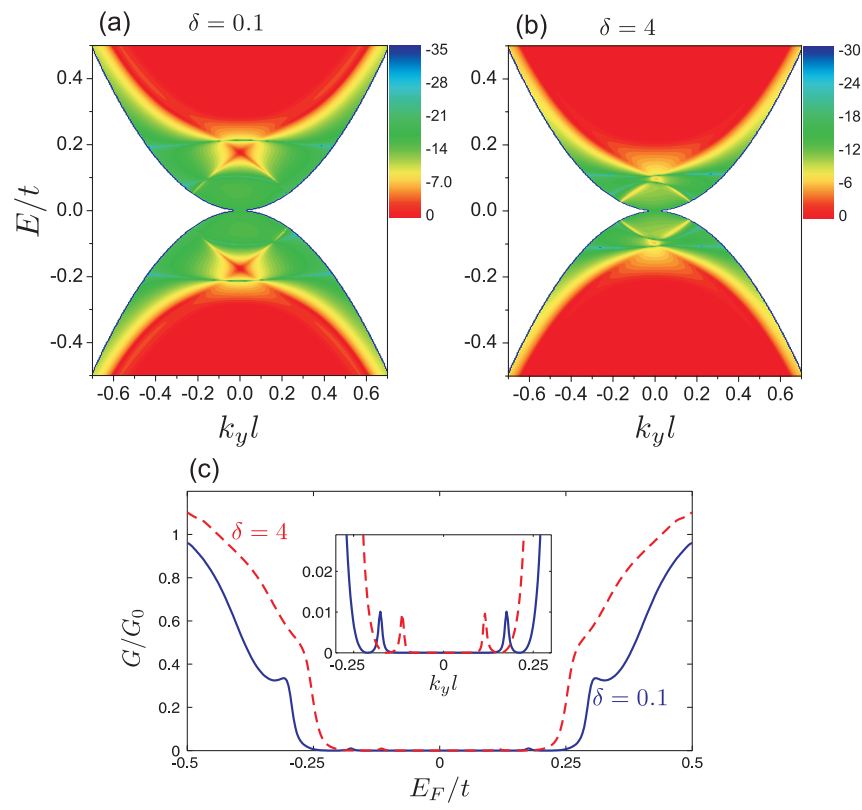

FIG. 20. (Color online) (a), (b) Contour plot of the transmission $T$ (in logarithmic scale) through a kink-antikink structure with the length $L=24(\approx 40 \mathrm{~nm})$ for $u_{b}=0.25$ and $d=6$ with (a) $\delta=0.1$ and (b) $\delta=4$. (c) Conductance $G / G_{0}$ vs Fermi energy of a kinkantikink potential for the same parameters as (a) and (b). 
Fig. 20(b). A small region of transmittance appears in the energy gap due to the chiral states that appear as small peaks in the conductance [see the inset of Fig. 20(c)].

\section{B. Magnetic field dependence}

Figure 15(b) shows the kink-antikink energy levels in the presence of an external magnetic field $(B=3 \mathrm{~T})$. The results show a shift of the four intragap energy branches as the magnetic field increases. In addition, the continuum of free states at zero magnetic field [shadowed region in Fig. 15(a)] is replaced by a set of Landau levels. The spinor components and probability densities associated with the points indicated inside the circle in Figs. 15(b) are shown in the panels (1b, 2b, $\ldots, 5 b)$. For nonzero magnetic field, the states show a shift of the probability density toward the region between the kink and the antikink. This is caused by the additional confinement due to the magnetic field.

The energy levels of a smooth kink-antikink profile (i.e., $\delta=10$ ) in the presence of a perpendicular magnetic field is presented in Fig. 16(b). Now, the crossing points (in the case of $B=0 \mathrm{~T}$ ) changed into anticrossings. In the inset of Fig. 16(b), an anticrossing is enlarged. Due to the strong confinement of the potential, the magnetic field can only lead to a shift up in energy of the localized chiral states. The wave spinors and the corresponding probability density for the points indicated by arrows in 16(b) are shown in the panels (1b, 2b, 3b, 4b). In the presence of an external magnetic field and at the crossing points of the topological states (panels 1b, 2b) due to the strong confinement by the potential, the magnetic field can only affect weakly the electrons. At the first anticrossing (panels $3 \mathrm{~b}$ and 4b), which arises from the overlap of the first bound states in the kink and antikink potentials, the electrons are confined closer to the center of the potential.

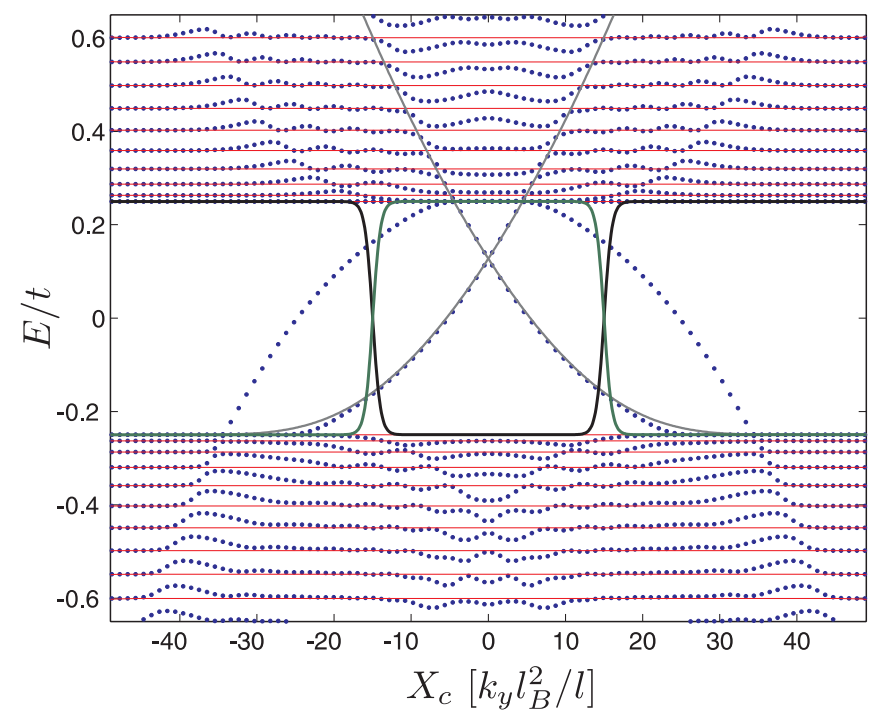

FIG. 21. (Color online) Energy spectrum of a kink-antikink profile in bilayer graphene as a function of the cyclotron orbit center $X_{c}$ with $u_{b}=0.25, d=15, B=7 \mathrm{~T}$, and $\delta=1$. Solid red lines correspond to the Landau levels of a biased BLG. The gray solid curves show fitted functions (given in the text) to the numerical results. The solid black and green curves describe the potential, respectively, in the upper and lower layers.
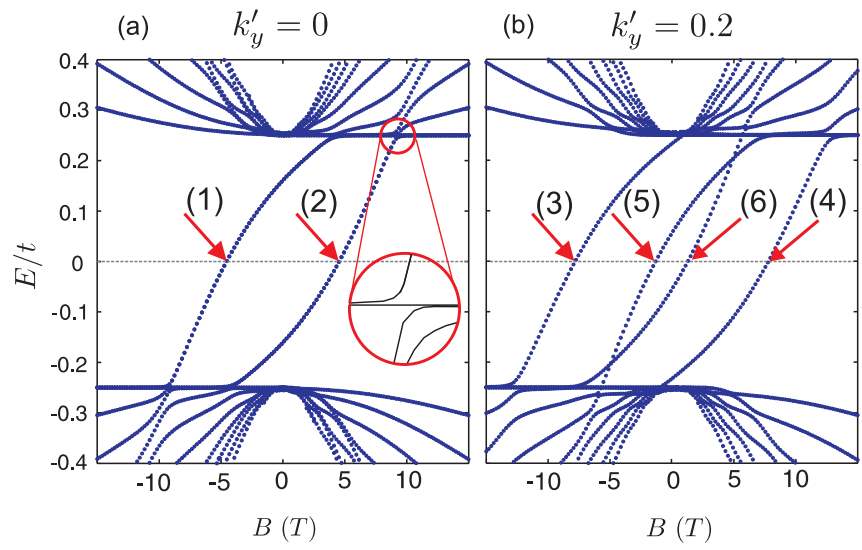

FIG. 22. (Color online) Energy levels of a sharp kink-antikink profile $\left(\delta=1\right.$ ) as a function of external magnetic field for $u_{b}=0.25$ with (a) $k_{y}^{\prime}=0$ and (b) $k_{y}^{\prime}=0.2$.

The energy levels for a sharp $(\delta=1)$ kink-antikink potential with $d=3$ is presented in Fig. 17(c). The crossings that appeared in the energy spectrum due to the overlap of the extra bound states in the absence of magnetic field [see Fig. 17(b)] now are replaced with anticrossings and the energy gap $E_{g}$ between the kink and antikink states is shifted up in energy due to the confinement by the magnetic field. Panels (1c, 2c, $3 c, 4 c)$ show the wave spinors and probability density for the points indicated by arrows in Fig. 17(c). The energy spectrum of a smooth kink-antikink potential with $d=3, \delta=10$ and in the presence of an external magnetic field $B=3 \mathrm{~T}$ is shown
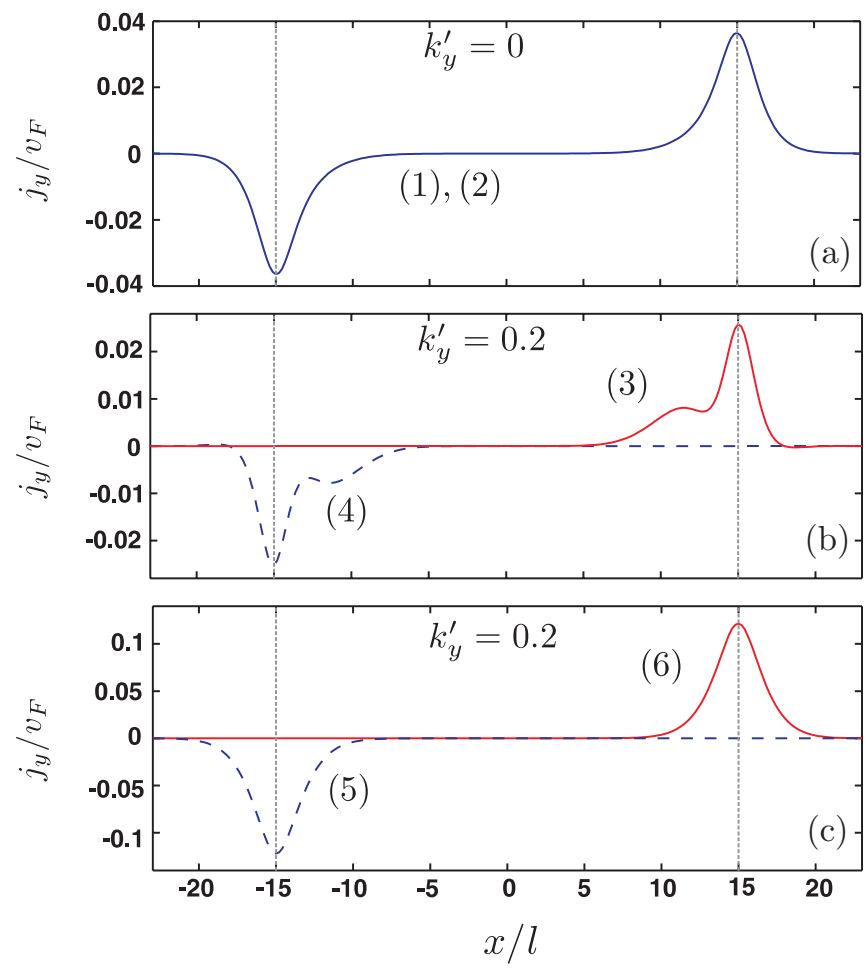

FIG. 23. (Color online) $y$ component of the persistent current of a kink-antikink profile in bilayer graphene as function of the $x$ direction for $E=E_{F}$ and for values of the magnetic field that are indicated by (1), (2), ... in Figs. 22(a) and 22(b). 

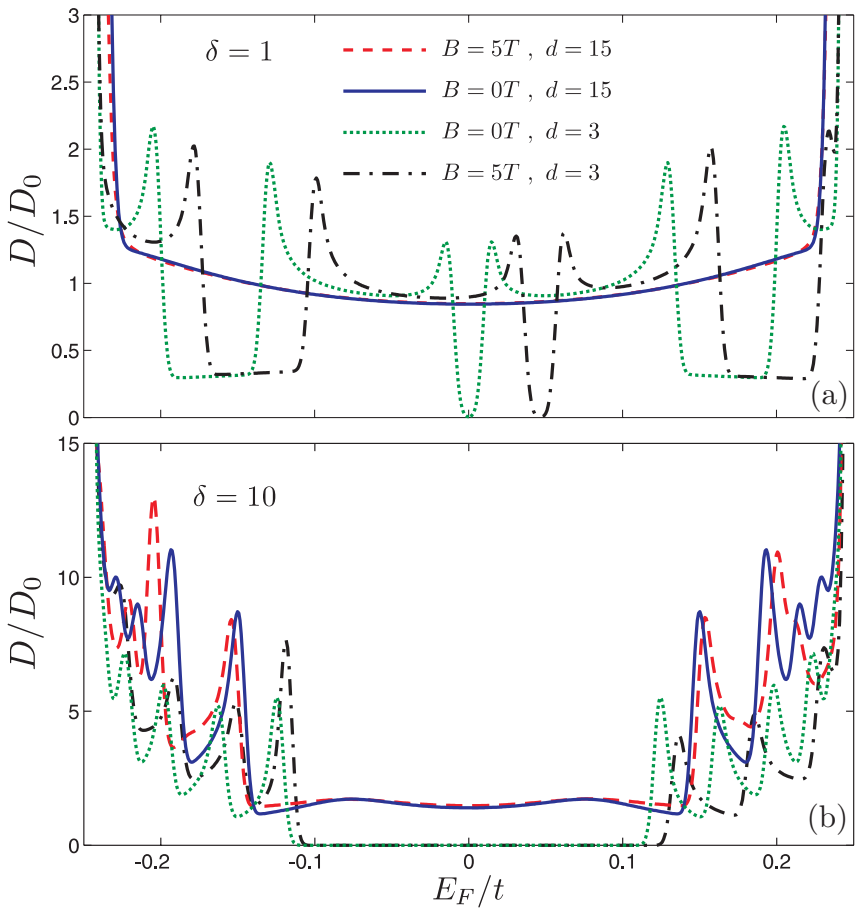

FIG. 24. (Color online) Density of states (DOS) of kink-antikink potentials for (a) $\delta=1$ and (b) $\delta=10$ with $u_{b}=0.25$. The results are presented for $d=3$ and 15 with $B=0$ and $5 \mathrm{~T}$.

in Fig. 18(b). In the presence of a magnetic field, the energy gap is shifted and the symmetry of the spectrum around $E=0$ for $B=0 \mathrm{~T}$ is broken [see Fig. 18(a)]. The energy gap in the presence of a magnetic field $(B=5 \mathrm{~T})$ is shown in Fig. 19 as red dashed curves. Notice that an external magnetic field only shifts up the energy gap, and the gap size remains constant.

The energy spectrum of a kink-antikink potential is shown in Fig. 21 as a function of orbit center $X_{c}$ for $\delta=1, u_{b}=0.25$, and $d=15$. The kink-antikink potential is depicted in the figure by the dashed curves. As for the single kink potential, the topological levels can be fitted to $\epsilon_{ \pm} \approx a \sqrt{\left(X_{c} \pm X_{0}\right)^{4}+\left(u_{b} / a\right)^{2}}$ (see gray solid curves) where $-(+)$ corresponds to the kink (antikink) branches ( $a=0.003$ and $X_{0}=41$ are the fitting parameters). Now, the Landau levels above the gap are affected by the kink-antikink potential, where anticrossing points appear along the topological levels. The solid red lines are the Landau levels in a biased BLG.

Figure 22 shows the dependence of the energies on the external magnetic field for (a) $k_{y}^{\prime}=0$ and (b) $k_{y}^{\prime}=0.2$. The branches that appear for $|\epsilon|>0.25$ correspond to Landau levels that arise from the continuum of free states. For the kink-antikink case, however, the overlap between the states associated with each confinement region allows the formation of Landau orbits. Therefore, the proximity of an antikink induces a strong dependence of the states on the external field.

Figure 23 shows plots of the $y$ component of the current density as function of $x$ for the states labeled (1) to (6) in Figs. 22(a) and 22(b). It should be noticed that a nonzero current can be found for $E=0$ and $k_{y}^{\prime} \neq 0$, as can be deduced from the dispersion relations. For $k_{y}^{\prime}=0$, the results presented in Fig. 23(a) show a persistent current carried by electrons localized at each kink region, irrespective of the direction of
$\boldsymbol{B}$, as exemplified by the states (1) and (2), which correspond to opposite directions of the magnetic field. For nonzero wave vector, however, as shown in Figs. 23(b) and 23(c), the current is strongly localized around one of the potential kinks. In Fig. 23(b), the current density curve shows an additional smaller peak caused by the strong magnetic field $(B \approx 7.5 \mathrm{~T})$, where the carriers can also be confined closer to the center.

The density of states of the topological states for (a) $\delta=1$ and (b) $\delta=10$ kink-antikink potential is shown in Fig. 24 with $d=3$ and 15 . The results show additional peaks for a sharp kink-antikink with $d=3$, which is due to the splitting of the topological levels. Note that the energy gap leads to a zero density at $E_{F}=0$ for zero magnetic field [green dotted curve in (a)], while shifting the gap in the presence of a magnetic field results in a nonzero DOS at $E_{F}=0$ [black dot-dashed curve in (a)]. For the smooth profiles, the nontopological 1D states lead to the appearance of additional peaks in the DOS [panel (b)] that shift with the magnetic field.

\section{CONCLUDING REMARKS}

In summary, we obtained the energy spectrum, the density of states, the transmission, and conductivity for carriers moving in BLG in the presence of asymmetric potentials (i.e., kink and kink-antikink profiles) in each layer of the BLG. Unidirectional chiral states are localized at the location of the kink (or antikink). By controlling the gate voltages and/or the smoothness of the kink profile, the number of one-dimensional metallic channels and their subsequent magnetic response can be configured.

The effect of an external magnetic field perpendicular to the bilayer sheet was investigated. We found that the influence of the magnetic field is very different for single and double kinks. Due to the strong confinement by the kink potential, the topological states are weakly affected by the magnetic field in the case of a single kink profile.

Changing the sign of the kink potential smoothly (i.e., broadening the kink potential) leads to extra bound states, which have a very different behavior as compared to the unidirectional topological states. First, these states are no longer unidirectional and they have a quasi-1D free-electron type of spectrum, which is asymmetric around $k_{y}=0$. Second, they are less strongly localized at the kink of the potential as compared to the chiral states, and their probability distribution appears as those of excited states of the chiral state.

In the case of parallel kink-antikink profiles, apparent crossings of the energy levels are found in the spectrum. Decreasing the distance between (and/or smoothing) the kinkantikink profiles turns into anticrossings. It opens a gap in the topological state spectrum. This allows for a robust 1D system having a tunable minigap.

\section{ACKNOWLEDGMENTS}

This work was supported by the Flemish Science Foundation (FWO-Vl), the Belgian Science Policy (IAP), the European Science Foundation (ESF) under the EUROCORES program EuroGRAPHENE (project CONGRAN), the Brazilian agency $\mathrm{CNPq}$ (Pronex), and the bilateral projects between Flanders and Brazil and the collaboration project FWO-CNPq. 
${ }^{1}$ R. Saito, G. Dresslhaus, and M. S. Dresselhaus, Physical Properties of Carbon Nanotubes (Imperial College Press, London, 1998).

${ }^{2}$ A. H. Castro Neto, F. Guinea, N. M. R. Peres, K. S. Novoselov, and A. Geim, Rev. Mod. Phys. 81, 109 (2009).

${ }^{3}$ X. Li, X. Wang, Li Zhang, S. Lee, and H. Dai, Science 319, 1229 (2008).

${ }^{4}$ T. Ohta, A. Bostwick, T. Seyller, K. Horn, and E. Rotenberg, Science 313, 951 (2006).

${ }^{5}$ E. McCann, Phys. Rev. B 74, 161403 (2006).

${ }^{6}$ E. V. Castro, K. S. Novoselov, S. V. Morozov, N. M. R. Peres, J. M.

B. Lopes dos Santos, Johan Nilsson, F. Guinea, A. K. Geim, and A. H. Castro Neto, Phys. Rev. Lett. 99, 216802 (2007).

${ }^{7}$ J. M. Pereira Jr., P. Vasilopoulos, and F. M. Peeters, Nano Lett. 7, 946 (2007).

${ }^{8}$ M. Zarenia, J. M. Pereira, Jr., F. M. Peeters, and G. A. Farias, Nano Lett. 9, 4088 (2009).

${ }^{9}$ I. Martin, Ya. M. Blanter, and A. F. Morpurgo, Phys. Rev. Lett. 100, 036804 (2008).
${ }^{10}$ M. Killi, T.-C. Wei, I. Affleck, and A. Paramekanti, Phys. Rev. Lett. 104, 216406 (2010).

${ }^{11}$ E. McCann and V. I. Fal'ko, Phys. Rev. Lett. 96, 086805 (2006).

${ }^{12}$ J. M. Pereira, F. M. Peeters, and P. Vasilopoulos, Phys. Rev. B 76, 115419 (2007).

${ }^{13}$ J. M. Pereira, F. M. Peeters, P. Vasilopoulos, R. N. Costa Filho, and G. A. Farias, Phys. Rev. B 79, 195403 (2009).

${ }^{14}$ L. Jing, J. Velasco Jr., P. Kratz, G. Liu, W. Bao, M. Bockrath, and C. N. Lau, Nano Lett. 10, 4775 (2010).

${ }^{15}$ I. Snyman and C. W. J. Beenakker, Phys. Rev. B 75, 045322 (2007).

${ }^{16}$ M. Ramezani Masir, P. Vasilopoulos, and F. M. Peeters, Phys. Rev. B 79, 035409 (2009).

${ }^{17}$ Michaël Barbier, Panagiotis Vasilopoulos, and François M. Peeters, Philos. Trans. R. Soc. A 368, 5499 (2010).

${ }^{18}$ M. Charbonneau, K. M. van Vliet, and P. Vasilopoulos, J. Math. Phys. 23, 318 (1982).

${ }^{19}$ Since the kink and antikink are close to each other, the height of the applied voltage to the layers is less than $u_{b}$ in the $\left|x^{\prime}\right| \leqslant d$ region, and we call the profile unperfect kink-antikink. 